\title{
WestVirginiaUniversity
}

THE RESEARCH REPOSITORY @ WVU

Graduate Theses, Dissertations, and Problem Reports

2010

\section{Electro-active liquid crystal dispersed composites}

Sarah E. Smith

West Virginia University

Follow this and additional works at: https://researchrepository.wvu.edu/etd

\section{Recommended Citation}

Smith, Sarah E., "Electro-active liquid crystal dispersed composites" (2010). Graduate Theses,

Dissertations, and Problem Reports. 2129.

https://researchrepository.wvu.edu/etd/2129

This Thesis is protected by copyright and/or related rights. It has been brought to you by the The Research Repository @ WVU with permission from the rights-holder(s). You are free to use this Thesis in any way that is permitted by the copyright and related rights legislation that applies to your use. For other uses you must obtain permission from the rights-holder(s) directly, unless additional rights are indicated by a Creative Commons license in the record and/ or on the work itself. This Thesis has been accepted for inclusion in WVU Graduate Theses, Dissertations, and Problem Reports collection by an authorized administrator of The Research Repository @ WVU. For more information, please contact researchrepository@mail.wvu.edu. 


\title{
Electro-Active Liquid Crystal Dispersed Composites
}

\author{
Sarah E. Smith
}

Thesis submitted to

the College of Engineering and Mineral Resources

at West Virginia University

in partial fulfillment of the requirements

for the degree of

\author{
Master of Science \\ in \\ Aerospace Engineering \\ Darran Cairns Ph.D., Chair \\ Kostas Sierros, Ph.D. \\ Wade Huebsch, PhD.
}

Department of Mechanical and Aerospace Engineering

Morgantown, West Virginia

2010

Key Words: Liquid Crystal, composites, polymerization, reactive mesogen 


\title{
Abstract \\ Electro-Active Liquid Crystal Dispersed Composites
}

\author{
Sarah E. Smith
}

In this thesis, the movement of anisotropic liquid crystal polymer rods in dispersion fluids of silicon oils was investigated. The rods demonstrated movement by an applied external AC field. The polymer rods used were $200 \mathrm{~nm}$ in diameter and $60 \mu \mathrm{m}$ in length produced using the template synthesis technique originally adopted by Shafran (2008). A reactive liquid crystal monomer was polymerized by ultraviolet light while the liquid crystal remained in the nematic temperature range. This curing permanently fixes the liquid crystal molecules, producing polymer rods independent of temperature. The rods were dispersed in silicon oils of viscosities 4.7, 9.2, and $99 \mathrm{cP}$, and injected into indium tin oxide coated glass cells.

An AC electric field was applied to the glass cells, resulting in multiple types of movement from the rods. In the silicon oil of viscosity $4.7 \mathrm{cP}$, the rod motions that were observed was spinning about a central point or large circular movements. In silicon oil of viscosity $9.2 \mathrm{cP}$, the motions that were observed was either wagging or flexing of the rods. Also, in the silicon oil of viscosity $99 \mathrm{cP}$, the motions that were observed were large circular movements and wagging. The ability of the polymer rods to respond in highly viscous dispersion fluids is technologically important for possible applications in active micromixers, morphing wings, and LC displays. 


\section{Acknowledgements}

I would like to thank my friends and family for their support.

I would like to thank Dr. Darran Cairns for being my advisor for the past two years. You gave me leadership throughout my career at WVU. Thank you for being so patient and understanding. Thanks to Dr. Kostas Sierros for helping me with the SEM and helping me to become better at scheduling and managing my time. I would like to thank my lab group for being supportive and for giving me help when I needed. Thank you.

I would like to thank my committee member Dr. Wade Huebsch, Dr. Darran Cairns, and Dr. Kostas Sierros for taking the time to read my thesis, evaluate my defense, and for all the corrections. 


\section{Table of Contents}

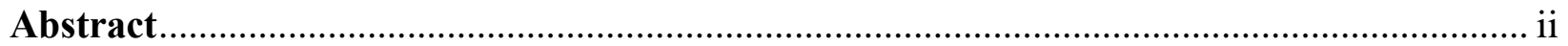

Acknowledgements ………………………….......................................................... ii

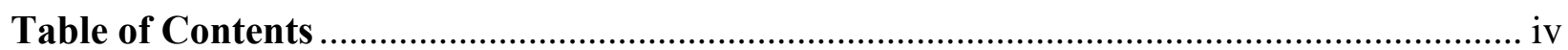

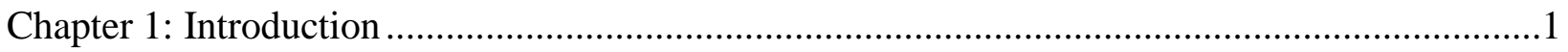

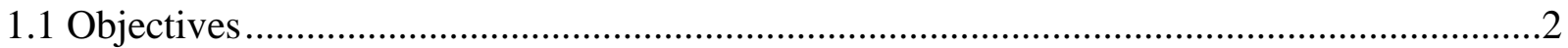

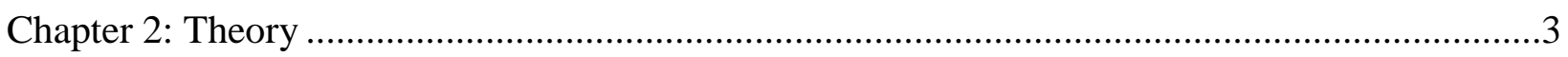

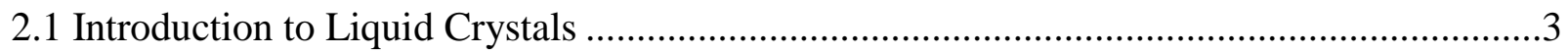

2.1.1 Mesophase Characteristics...................................................................................

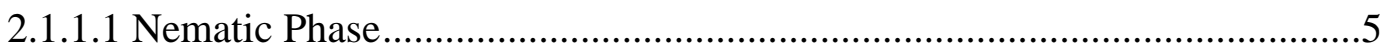

2.1.1.2 Cholesteric Phase …...........................................................................6

2.1.1.3 Semectic Phase (..............................................................................6

2.1.2 Orentational Order and Temperature ...................................................................

2.1.3 Electric and Magnetic Field Effect .....................................................................

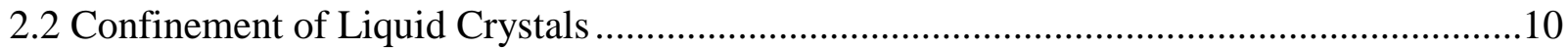

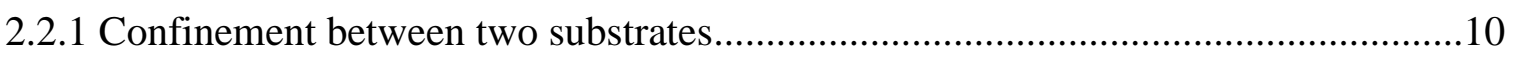

2.2.2 Confinement to Spherical and Elliptical Geometries.............................................11

2.2.3 Confinement to Cylindrical Geometries ..................................................................11

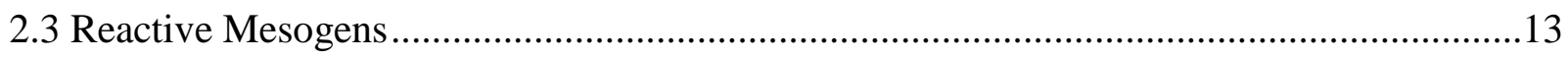

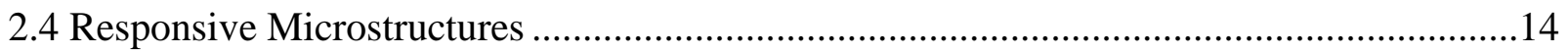

2.5 Polymer Dispersed Liquid Crystals.........................................................................15

2.5.1 Fabrication Methods .................................................................................19

2.5.1.1 Encapsulation ......................................................................................19

2.5.1.2 Phase Separation ...........................................................................19

2.5.1.2.1 Polymerization Induced Phase Separation...................................19

2.5.1.2.2 Thermally Induced Phase Separation.............................................20

2.5.1.2.3 Solvent Induced Phase Separation ................................................20

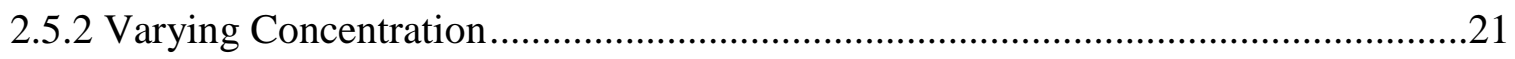

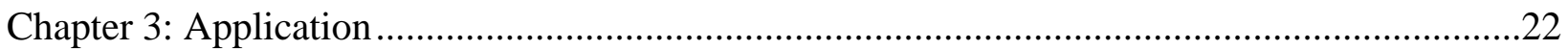

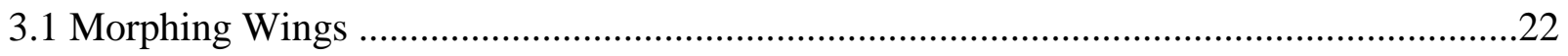

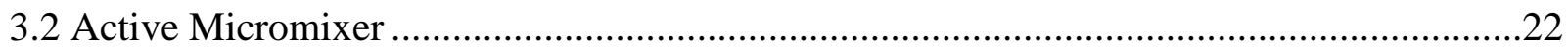




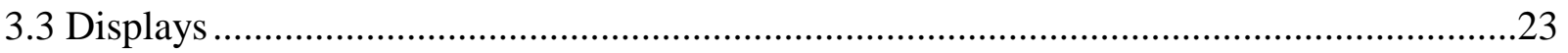

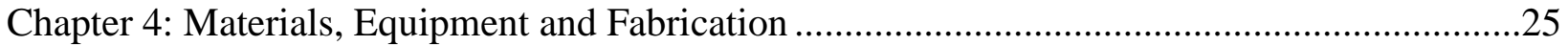

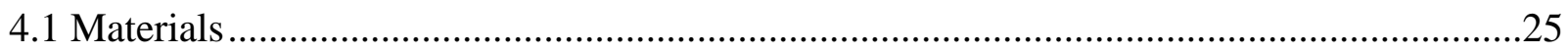

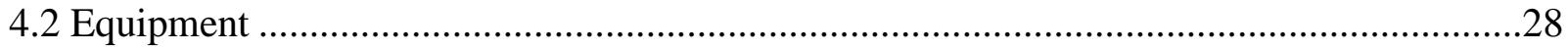

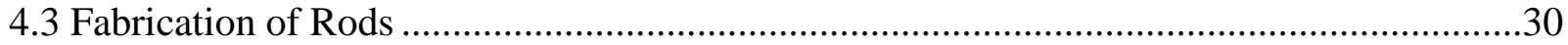

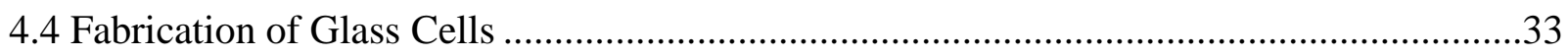

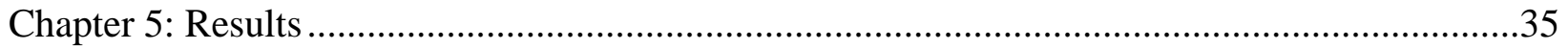

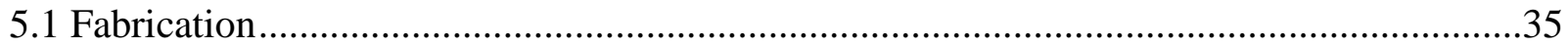

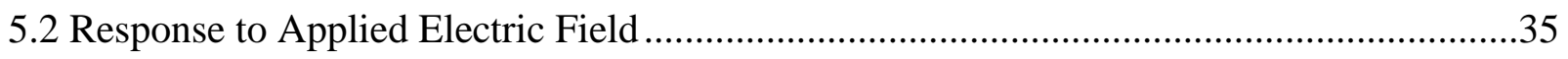

5.2.1 Low Viscosity Dispersion Fluid ............................................................................36

5.2.2 Medium Viscosity Dispersion Fluid ......................................................................

5.2.3 High Viscosity Dispersion Fluid.............................................................................

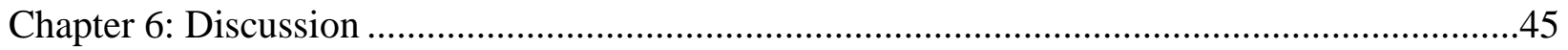

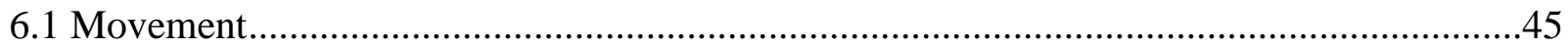

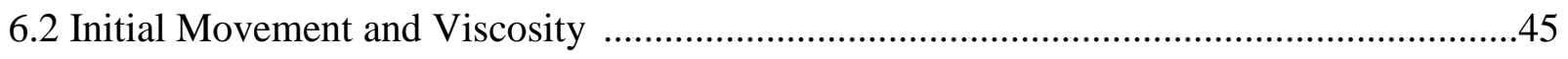

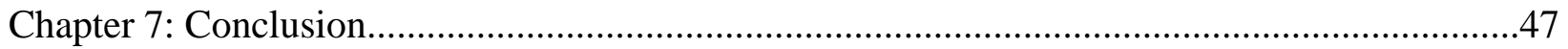

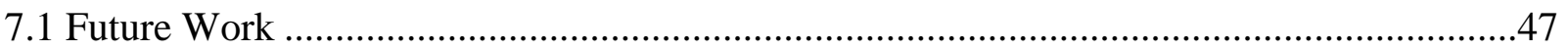

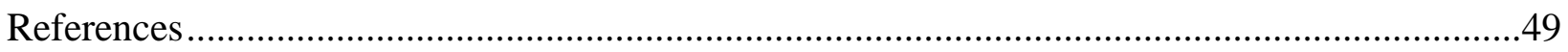




\section{Chapter 1: Introduction}

Confinement of liquid crystals to create a reactive microstructure has become a popular area of research. Since liquid crystals have unique properties, their structures are affected by small changes, thus allowing new and exciting research developments. The anisotropy, referring to varying properties when measured on different axes, is a unique characteristic of liquid crystals that allows them to be good candidate for reactive microstructures. Based on the conditions in which the reactive microstructures are produced, their properties may be different. Those properties include electrical, ${ }^{[1]}$ mechanical, ${ }^{[2]}$ optical, ${ }^{[3-5]}$ or the final shape of the reactive microstructure. ${ }^{[6-8]}$ Responsive microstructures produced by

Cairns ${ }^{[9]}$ laid the groundwork for this project. Cairns and coworkers were able to make solid anisotropic polymer spheres from liquid crystal monomers or reactive mesogens. The polymer spheres were dispersed in glycerol and then exposed to an ultraviolet light. By curing the liquid crystal droplets, while in the nematic state, captured the bipolar director field. Curing with UV light allowed the liquid crystal nematic phase to be perfectly preserved. This created an induced dipole within the droplets. Once an electric field is applied, the bipolar director field of the droplets aligns with the electric field. This alignment of all the droplets allowed light to pass through the film.

This thesis was initiated by the work of Cairns ${ }^{[10]}$ who developed rod-like structures using anopore membranes as a method to confine liquid crystals. To expand further this idea, responsive microstructures have been created using anopore membranes as a method to confine liquid crystals. Using these rod-like structures, dispersed in viscous liquids, cells will be created that can be manipulated with an applied electric field. 


\subsection{Objectives}

The main objectives of this research include:

- Fabrication of liquid crystal rods dispersed in viscous liquids

Fabrication of the liquid crystal rods is the first step in this research. The rods must then be evenly dispersed within viscous liquids.

- Application of electric fields to cause movement of rods

Once the rods have been fabricated and evenly dispersed within the viscous liquids, it is then necessary to cause movement of the rods.

\section{- Classification of movement}

After movement of the rods is achieved, this movement will be classified.

It will be shown that the rods are responsive, within viscous liquids, when electric fields are applied. A variety of movements will be documented in low, medium, and high viscous liquids. 


\section{Chapter 2: Theory}

\subsection{Introduction to Liquid Crystals}

Liquid crystals have attracted much attention since 1888 when they where first discovered by Reinitzer who observed cholesteryl benzoate having two melting points. ${ }^{[1]}$ The first melting point is observed when it melts to a cloudy liquid and the second is observed when the liquid becomes transparent.

Liquid crystals are a mesophase, observed between the solid and liquid state ${ }^{[1]}$ that will retain properties from both the solid state and the liquid state. They exhibit more orientation order than a liquid and less than a solid crystal, which is shown in Figure 2.1.1.

In addition, liquid crystals on average will align with each other; they do not exhibit strict parallel arrangement but rather show group-favored orientation. ${ }^{[12]}$ The molecules of the liquid crystals are typically elongated in shape, depicted as a rod shape. Physically they are typically 20-30 ̊̊ long and $\sim 5 \AA$ wide. This phenomenon occurs because of van der Waals forces. In a liquid crystal molecule, the electrons are mobile and some will be slightly positive and slightly negative. As molecules interact, a temporary dipole will cause an induced dipole in another molecule. This process continues and dispersion forces or van der Waals forces cause a groupfavored orientation. ${ }^{[13]}$ 


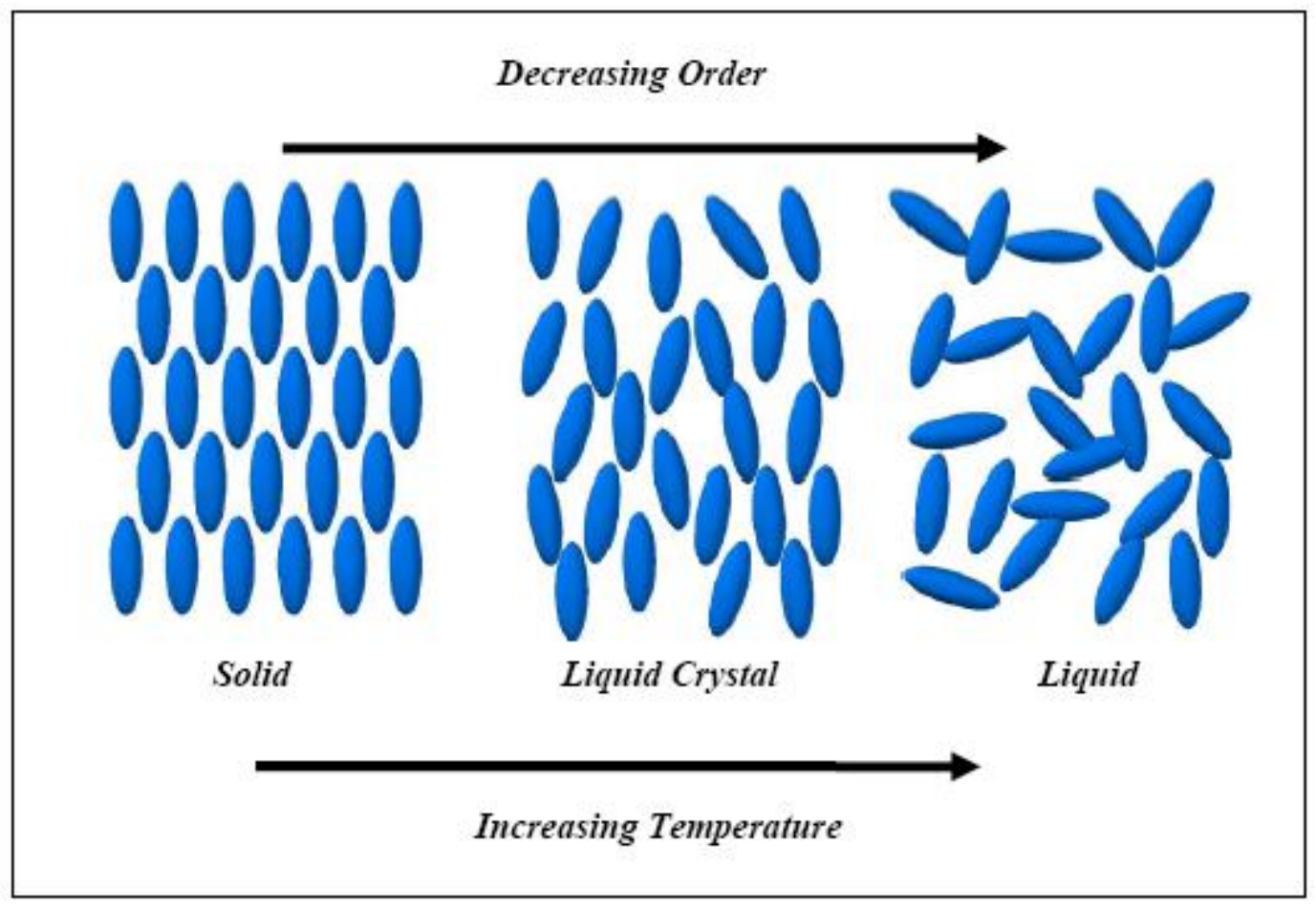

Figure 2.1.1: The order of liquid crystal molecules decrease as temperature increases ${ }^{[14]}$

\subsubsection{Mesophase Characteristics}

The liquid crystal phase is often called the mesophase, and a substance having such character a mesogen. ${ }^{[15]}$ The molecules are typically long and rod-like in shape, and the large anisotropy of the molecule is essential for the appearance of the mesophase. There are two groups of liquid crystals that will be discussed; thermotropic (i.e. liquid crystals are formed by a change in temperature) and lyotropic (i.e. liquid crystals phases that are formed because of the addition of a solvent).

As mentioned above, the molecules of a liquid crystal are long. In the liquid crystal phase, the molecules tend to align parallel to each other along a certain direction. There are also additional structures present and these are divided into three different phases: nematic, cholesteric, and smectic. These phases can be seen in Figure 2.1.1.1. 


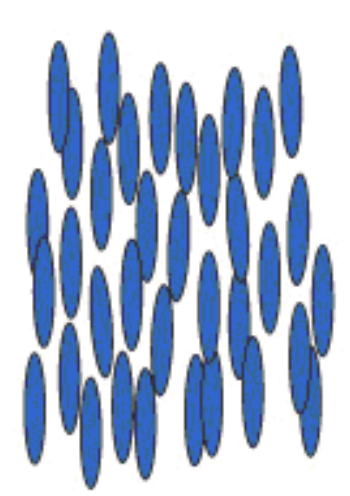

Nematic

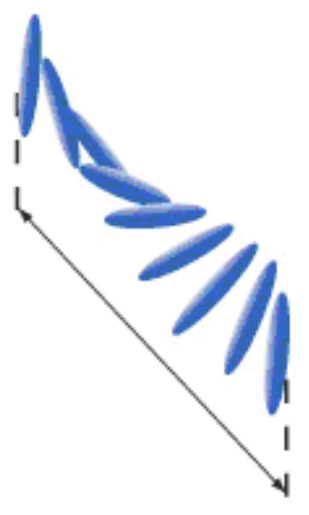

Cholesteric

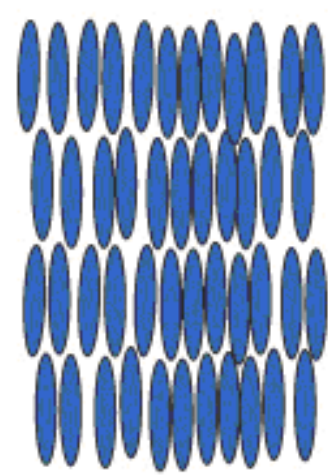

Smectic

Figure 2.1.1.1: Three phases based on molecular arrangements ${ }^{[16]}$

The type of liquid crystal that will be used is a reactive mesogen. Reactive mesogens or liquid crystal monomers are photo-polymerizable and form densely cross-linked polymer networks when irradiated

with UV light. ${ }^{[17-19]}$ The procedure permanently "freezes" the molecular orientation of the liquid crystal, forming a rigid polymer structure, which is independent of temperature after curing. ${ }^{[17-19]}$

\subsubsection{Nematic Phase}

In the nematic phase, the molecules are parallel to each other but their distribution is random. ${ }^{[20]}$ Therefore, there is an orientational order and no positional order, but the molecules are able to move around from one region to another and around their axis. ${ }^{[20]}$ 


\subsubsection{Cholesteric Phase}

In the cholesteric phase, the molecules are parallel to each other but are twisted perpendicularly to the director, creating a helical structure. ${ }^{[20]}$ These substances are in parallel sheets or layers, which can be seen in Figure 2. An important characteristic of cholesteric mesophase is the pitch. When the molecules undergo a full $360^{\circ}$ twist, this is known as the chiral pitch and this twisting repeats itself every half turn or from $0^{0}$ to $\pm 180^{\circ}$. The twisting or pitch within the molecules can reflect light of wavelengths equal to the pitch length. A color can be reflected when the pitch is equal to the corresponding wavelength of light in the visible spectrum. ${ }^{[20]}$ The chiral pitch changes as the temperature or composition changes. For example, if the temperature is increased, angle at which the director changes can be made larger, and thus tighten the pitch.

\subsubsection{Smectic Phase}

The molecules are parallel to each other and normal to the layer plane in the smectic phase. [14,20] These well-defined layers or regularity in the distribution pattern can move past one another or rotate relative to each other. Since the smectic phase possesses well-defined layers, different direction of alignment relative to the layer plane creates sub-classifications. " ${ }^{20]}$ "In the Smectic A phase, the axis is normal to the layers, in the B phase the molecules have a hcp (hexagonal closed packed) structure within the layers, in the $\mathrm{C}$ phase the axis is tilted, and in the D phase the structure is cubic." ${ }^{[20]}$ 


\subsubsection{Orientational Order and Temperature}

There is a preferred direction in which the molecules in the liquid crystal phase tend to align and this is referred to as the orientational order. Since the molecules are not fixed, the order is averaged and is defined by a Director that points in the preferred direction. The Director is a unit vector that points along a specific direction in space and is a function of the position vector. An example of a Director can be seen in Figure 2.1.2.1. To specify the degree of order, the orientational function $\mathrm{S}$ can be used. $\mathrm{S}$ is described as follows:

$$
S=\frac{3 \cos ^{2} \theta-1}{2}
$$

Where $\theta$ is the angle between the axis of a typical molecule and the Director. The order function can range from 0 to 1 , but will often lies between 0.3 to 0.9 , where 0 refers to no order (complete disorder, like an isotropic liquid), and 1 refers to complete order (like a solid). ${ }^{[20]}$

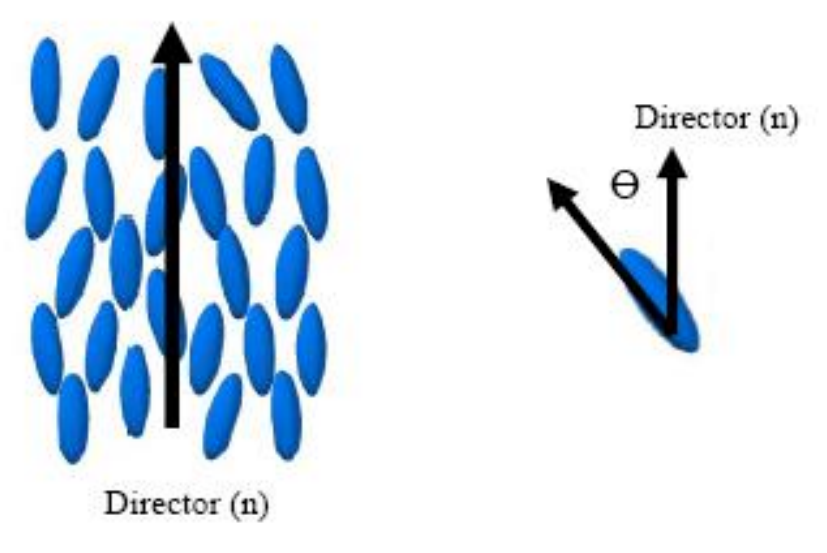

Figure 2.1.2.1: An example of a Director ${ }^{[14]}$ 
Temperature is an important contribution factor in liquid crystal orientational order. As temperature increases, the order decreases. One can see from Figure 2.1.2.2 that the orientational order or order parameter S falls sharply at the clearing point of the liquid crystal or the start of the isotropic liquid phase. At temperatures above the clearing point, the liquid crystal no longer behaves as such and acts more like a liquid.

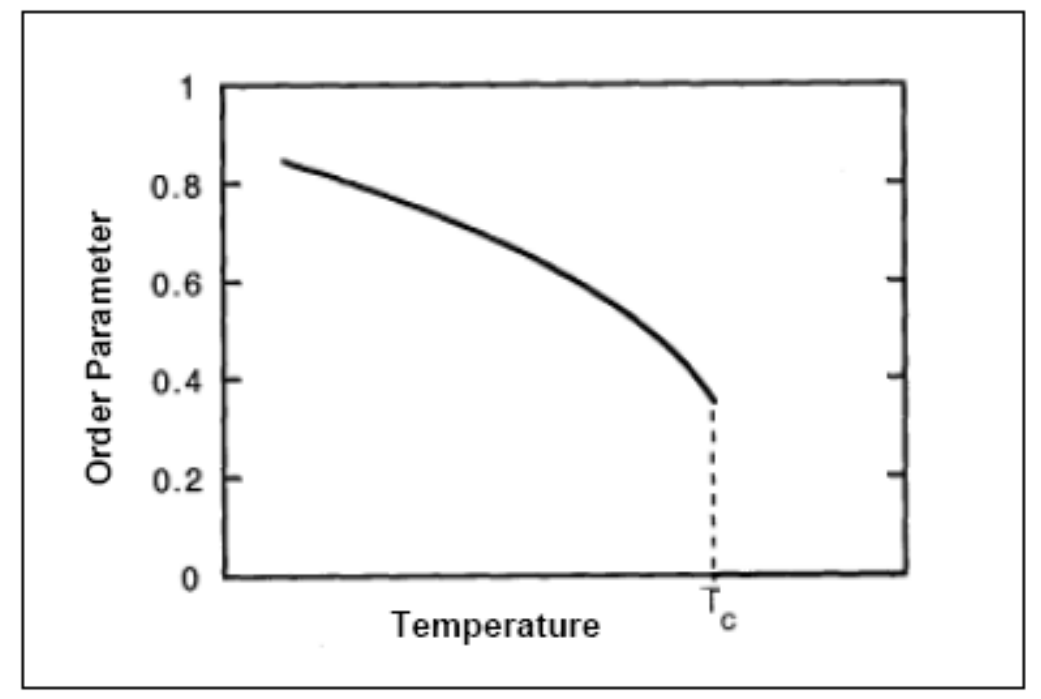

Figure 2.1.2.2: The order parameter falls sharply as the clearing temperature is reached ${ }^{[20]}$

\subsubsection{Electric and Magnetic Field Effect}

An electric or magnetic field produces important effects in the liquid crystals. When an electric field is not applied to permanent dipole liquid crystal molecules, will have an equal chance to point North or South. However, when an electric or magnetic field is applied, the liquid crystal molecules orientate their polls with respect to the field and this deformation can be described as splay, twist or bend. An artistic representation of splay, twist and bend can be seen in Figure 2.1.3.1. Based on the amount of order that is present, the phase can be determined. An example of this principle, using an electric field, is in liquid crystal displays (LCDs). The 
majority of molecules in the nematic phase will have a permanent or induced electric dipole. ${ }^{[20]}$ Once an electric field is present, the molecules will rotate until their long axis is aligned with the applied field. ${ }^{[20]}$ As the electric field intensity increases, so does the force causing the molecule to rotate. If many molecules are contained and the Directors are pointing in the same direction, the dipoles of each molecule can add together to form a large electric dipole. ${ }^{[20]}$ Magnetic fields can also produce similar effects in liquid crystals that are diamagnetic.

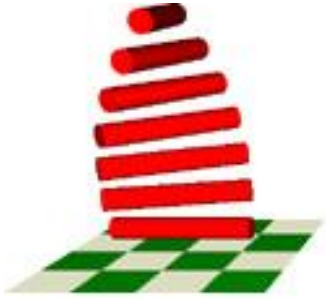

Twist

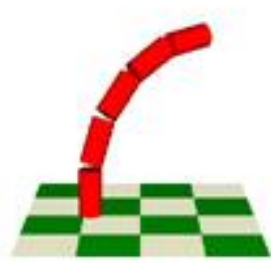

Bend

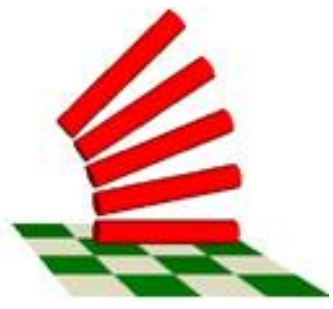

Splay

Figure 2.1.3.1: An artistic representation of splay, bend and twist ${ }^{[21]}$

In polymer dispersed liquid crystals, with sufficiently high magnetic fields and under strong anchoring conditions liquid crystal droplets deform into anisotropic particles. Therefore, the bulk of Directors aligns along the imposed field but maintain orientation along the axis of the polymer-liquid crystal interface. ${ }^{[22]}$ This allows the creation and control of the shape and director textures in polymer dispersed liquid crystals by using a magnetic or electric field. ${ }^{[22]}$

Also in polymer-dispersed liquid crystals, where the polymer was chemically modified, the splay and bend was obtained from TEM images of the director field, resulting in large differences in elastic anisotropy. ${ }^{[23]}$ Thus showing that small chances in the chemical composition can have large effects on the splay, twist and bend of the liquid crystal molecules within the polymer. 


\subsection{Confinement of Liquid Crystals}

Since liquid crystals are able to flow like a liquid, it is necessary that they are confined. Usually, the liquid crystals are sandwiched between two flat substrates, for example as in a LCD display. ${ }^{\text {[24] }}$ Along with confining liquid crystals, orientation can be controlled by inducing alignment. By rubbing or scratching the substrate, alignment of liquid crystals will occur once they are placed on the substrate. If a surface is coated with a material, which naturally aligns, the liquid crystals will follow suit and align on the substrate was well. Finally, orientation can be controlled by externally applying a magnetic or electric field. By confining the liquid crystals, the molecular architecture is changed and their mechanical and responsive properties will be different from their bulk state. ${ }^{[25]}$

\subsubsection{Confinement between Two Substrates}

In a study of nematic liquid crystal placed between two glass cells, an electric field was applied, and a switching memory effect was determined. The memory effect was a reflection of the electric displacement, being a function of both the applied field and the materials properties adjusting to the value of the field applied. ${ }^{[25]}$ The memory effect with the liquid crystals became evident when the voltage was changed abruptly. ${ }^{[25]}$

Bi-stable nematic liquid crystals can be aligned by using surface anchoring between two aligning films of $\mathrm{SiO} .{ }^{[26]} \mathrm{SiO}$ was used since it is known to induce alignment in bulk liquid

crystal layers. ${ }^{[27]}$ A simple mode of the system, by the elastic term coming from the topography of the $\mathrm{SiO}$ layer, can be found in reference 27. The independent anchoring of each source is obtained by means of a composite surface made with a film of photopolymer on an anisotropic SiO layer. 


\subsubsection{Confinement to Spherical and Elliptical Geometries}

Uniformly aligned liquid crystals can be produced without any surface aligning of layers.

[28] By confining liquid crystals in spherical or elliptical geometries, their properties change

because of a high surface to volume ratio. ${ }^{[29]}$ The polymer orientation as well as the shape anisotropy influences the liquid crystal alignment when confined. ${ }^{[30]}$

Liquid crystal droplets can also be used to induce alignment. Optoelectronic devices were fabricated by $\mathrm{Wu}^{[31]}$ from $\mathrm{CdS}$ semiconductor nanorods and nematic liquid crystals. Cells were created by rubbing ITO glass, which induced parallel alignment of the liquid crystal. Once an electric field was applied, the liquid crystal realigned to perpendicular alignment with respect to the ITO glass and this caused the CdS rods to align.

\subsubsection{Confinement to Cylindrical Geometries}

Liquid crystals confined to cylindrical cavities have been of interest since the early 1970s. ${ }^{[30]}$ Different types of cylindrical cavities can be used to confine liquid crystals and those include: anopore membranes, ${ }^{[32,33]}$ Aerogels, ${ }^{[29]}$ Vycor glass, ${ }^{[29]}$ nuclepore membranes ${ }^{[29]}$ and carbon nanotubes. ${ }^{[34]}$

Anopore membranes are the interest in this research since preliminary research by Cairns has concluded that liquid crystal rods can be produced by anopore membranes. An anopore membrane is constructed by the anodic oxidation of aluminum and has a porosity of $40 \% .{ }^{[34]}$ The membranes are available in multiple sizes ranging from .02 to .2 microns in diameter with a thickness of 60 microns. ${ }^{[34]}$ In Figure 2.2.3.1, a Scanning Electron Microscope (SEM) image of a 0.2-micron anopore membrane can be seen. 


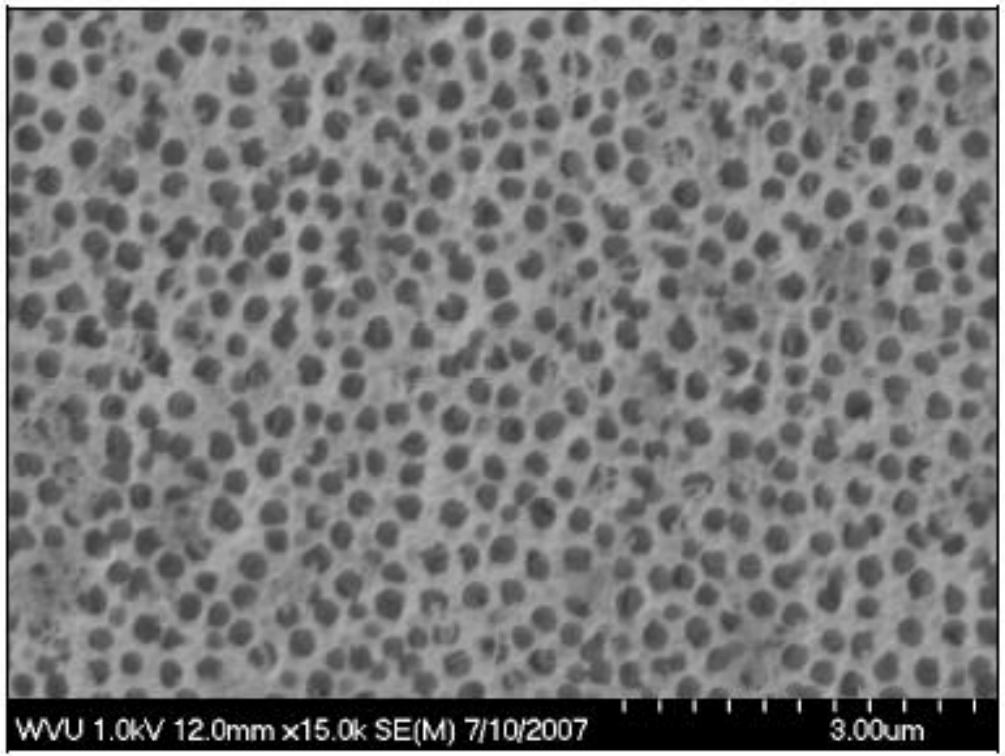

Figure 2.2.3.1: "SEM image of an anopore membrane with a diameter of 0.2 microns" 15

Multiple approaches of determining the molecular dynamics and anchoring effects of cylindrical cavities of anopore membranes filled with liquid crystals have been studied at cavities above and below $1 \mu \mathrm{m} .{ }^{[35]}$ Nuclear Magnetic Resonance (NMR) is one of those techniques. Developed in the early 1950s, it can provide one of the most accurate methods for determining molecular structure and has been used to reveal the orientational order of liquid crystals confined to cylindrical cavities and anopore membranes. ${ }^{[35-38]}$ Possible director fields are shown in Figure 2.2.3.2. In this thesis, the intended director field is where the liquid crystal molecules are aligned parallel to the cylinder. This would allow the molecules to sum together and create one large dipole. 


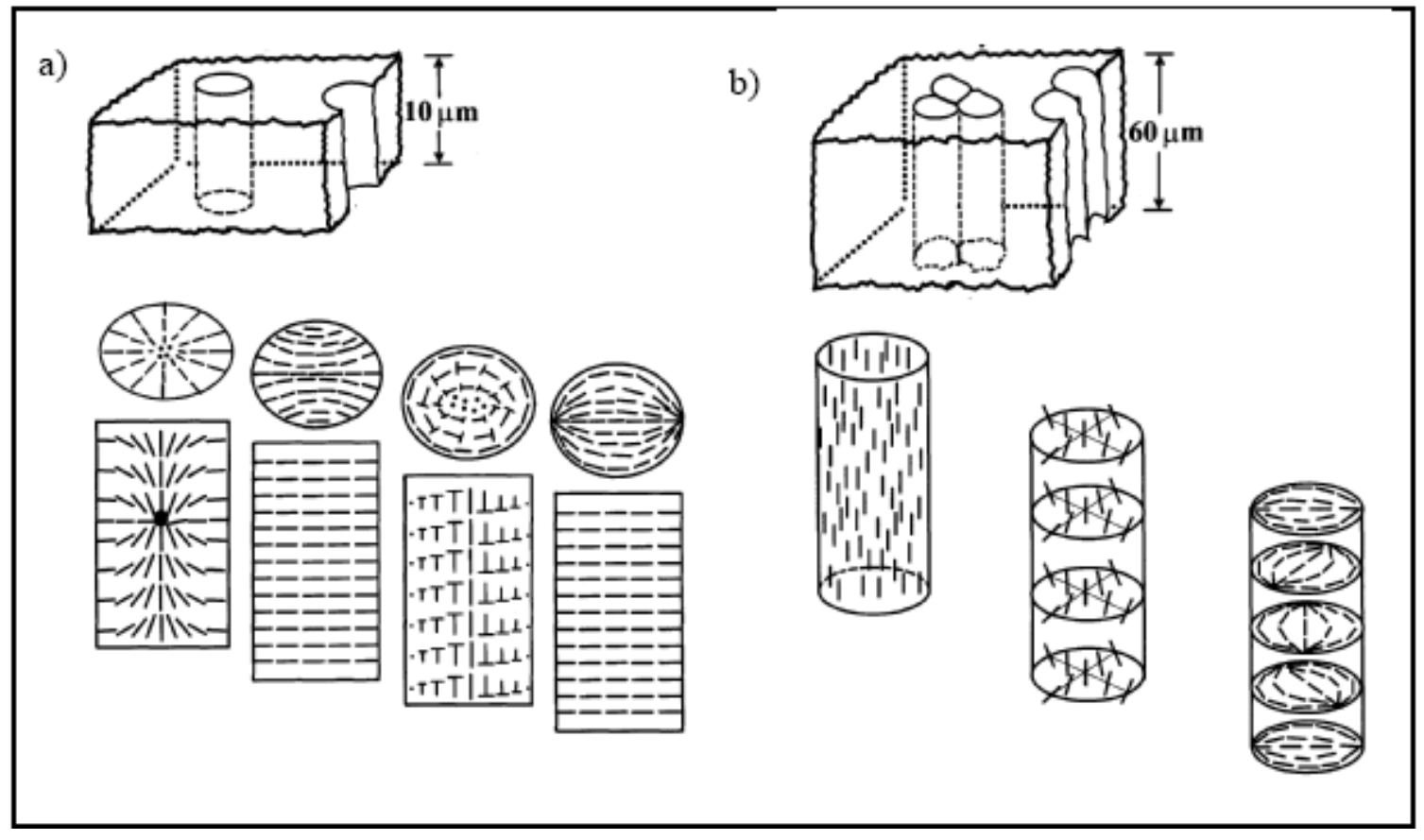

Figure 2.2.3.2: Possible director fields of liquid crystals in cylindrical confined geometries ${ }^{[30]}$

\subsection{Reactive Mesogens}

Reactive mesogens are photo-polymerizable liquid crystals that form densely crosslinked polymer networks when they are cured with UV light. ${ }^{[39]}$ Reactive mesogens are used throughout this research. Cross-linking permanently freeze the molecular orientation of the liquid crystals, forming a rigid polymer structure that is independent of temperature after curing.

[39] Because of this characteristic, the liquid crystal molecules, before or during polymerization, can be manipulated by an external applied field, photo-masks or surface treatments to modify the molecules: thus, creating liquid crystal molecules that meet specific needs. After polymerization the modification created prior, can be locked in after polymerization. This can lead to multiple applications including thermally reactive polymer films, ${ }^{[40-42]}$ geometrical and optical anisotropic colloids, and responsive microstructures for electro-optical switches. 


\subsection{Responsive Microstructures}

Cairns also developed rod-like structures using Anopore membranes as a method to confine liquid crystals. The work of this thesis was initiated with the inspiration that the polymer dispersed anisotropic liquid crystal films can be manipulated with an applied electric field. It was also concluded by Cairns that the rotation speed of the spheres was found to be dependent on field strength, viscosity and director field alignment.

Magnetically activated rod arrays, produced by Evans and coworkers, ${ }^{[43]}$ and may be useful in sensing applications as well as in microfluidics. Nano and micro rods were fabricated from poly(dimethylsiloxane) (PDMS) and iron oxide particles. They attached one end of the rods to a substrate, and the opposite end was free to move. Once a magnetic field is applied, the rods rotate and move back and forth. A magnetically operated switch has also been produced by Dierking. ${ }^{[43]}$ Nematic liquid crystals and single wall carbon nanotubes were used. In the OFF position, the nanotubes and liquid crystals are parallel to an ITO substrate and in the ON position; the nanotubes rotate perpendicular to the ITO substrate, creating conductive nanotubes that complete the connection between two ITO substrates.

Liquid crystals can also be used to force alignment in the application of optoelectronic devices. ${ }^{[44]} \mathrm{Wu}^{[45]}$ used CdS semiconductor nano rods and nematic liquid crystals to induce alignment on the CdS nano rods. By rubbing ITO glass, parallel alignment of the liquid crystal is induced and when an electric field is applied, the liquid crystals align perpendicular, which caused the $\mathrm{CdS}$ rods to align with the liquid crystals.

Liquid crystal droplets have been shown to have sub-ms switching times in an optical switch application. ${ }^{[46]}$ In this example, the torque of optically trapped nematic droplets was 
measured from circularly polarized light. Wood ${ }^{[47]}$ experimentally characterized which properties of liquid crystal droplets had the largest effect on the transfer of optical torque. The strength of the light intensity was proportional to the amount of torque induced on the liquid crystal droplet, where the highest intensities gave the largest torque values. $2.5 \mu \mathrm{m}$ and $8.0 \mu m$ droplets gave the highest drag torque values which were attributed to wave plate-like behavior.

The alignment of rod shaped gold particles in an aqueous dispersion with an applied electric field has also been demonstrated. ${ }^{[48]}$ The orientation order of the rods was determined by the absorbance spectra of the rod dispersions in an aqueous solution as a function of the electric field strength.

In addition, by using glancing angle deposition and porous chiral thin films, a reversible electro-optic switch of the liquid crystal component has been demonstrated. ${ }^{[49]}$ The pores have been embedded with the liquid crystals to produce a new type of optical material. These films have been shown to induce chiral alignment in nematic liquid crystals that do not form chiral phases on their own, resulting in an enhanced chiral optic response of the nematic liquid crystals. The chiral optic response vanishes with the application of an electric field; these films are envisioned for the application of displays.

\subsection{Polymer Dispersed Liquid Crystals}

Polymers are composed of monomers, which are their basic unit that repeats in a long chainlike sequence. Because of the repeating sequence, polymers exhibit distinctive properties based on the ability to orient the chains in a useful manner. The bonds that hold the monomers together are typically covalent or ionic in nature and are therefore strong. The degree of polymerization is known as the number of monomers within a polymer chain and is typically > 
$10^{4}$. Polymers are grouped according to the amount of crosslinking and what is crosslinked with the polymer. The more crosslinking a polymer possesses, the stronger it becomes at room temperature, but this makes the polymer more brittle.

In the 1980s Polymer Dispersed Liquid Crystals were first investigated as a new composite, which was the first major application of polymer liquid crystal spheres. ${ }^{[50]}$ PDLCs are constructed from liquid crystals bound in a polymer film. ${ }^{[50]}$ They are a composite material in which liquid crystals are randomly distributed in a polymer matrix. PDLCs most popular application is electro-optical devises; one of which is a privacy window that can be switched between transparent and opaque depending on an applied electric field. ${ }^{[50]}$ PDLCs are fabricated by phase separating the liquid crystal from the polymer or by encapsulation. ${ }^{[50]}$ Depending on the method chosen, the properties of the PDLCs will be different. These properties include the size and morphology of the liquid crystal droplets and the heating and cooling rates involved in the production of PDLCs.

Polymer dispersed liquid crystal films reflect a switching memory that is attributed to the applied field as well as the material's properties need for some finite time to adjust to the applied electric field. ${ }^{[51]}$ This memory effect can be seen when the voltage is changed abruptly and is a function of the present and past electric field and the present and past director. ${ }^{[52]}$

In PDLC thin films, near-field scanning, and optical microscopy was used to investigate field and frequency dependent molecular reorientation. ${ }^{[53]}$ It was concluded that using near-field methods, molecular reorientation processes maybe induced and detected in highly localized regions of the materials. In addition, it was noted that stronger fields were required to reorient the liquid crystals near the polymer interface when compared to those required to reorient the 
droplet center. Therefore, different reorientation mechanisms exist along the axis of the liquid crystal droplets. ${ }^{[53,54]}$

PDLC materials with nano-sized droplets have also been characterized. ${ }^{[55]}$ The nanosized droplets of liquid crystal, ranging from 100-250 nm, were prepared by rapid polymerization of a highly functionalized monomer. By controlling the polymerization rate and adjusting the concentration of the liquid crystal within the PDLC material, different types of sample morphologies were obtained. It was reported that the electro-optic properties of the PDLC films strictly depend on the sample morphology. ${ }^{[55]}$ The ratio between liquid crystal and polymer concentration was an important parameter in determining the electro-optic properties of the films.

Ten different liquid crystal monomers were synthesized to be polymerized into sphere. [56] When in the presence of a polarized light, some of these solid spheres could be rotated. Also, various dilutions and mixtures of the liquid crystal monomers where characterized to find the typical diameter. Garces-Chavez ${ }^{[57]}$ also fabricated spheres using UV photo-polymerizing liquid crystals. They found that the spheres rotation increased linearly with light beam power.

Anisotropic colloids have been produced by Fernandez-Nieves by mechanically deforming reactive monomer bipolar droplets. The droplets were suspended in a polyvinyl alcohol (PVA) film and were stretched (uniaxially) to 12 times their original diameter and then were photo-polymerized. After dissolving the PVA matrix, liquid crystal ellipsoids were the resulting structure.

Stretched polymer dispersed liquid crystal films were characterized by Amimori to determine the relationship between polarization and uniaxial stress-strain properties. It was 
determined that the liquid crystal alignment was dependent on both shape anisotropy within the PVA matrix and the polymer orientation while the films were being stretched. Amimori ${ }^{[58]}$ also stretched uniaxially bipolar droplets and observed them with deuterium nuclear magnetic resonance. The results of the spectra confirmed that the liquid crystal ellipsoids had a bipolar director field and the liquid crystals aligned with the stretched axis.

PDLC technologies can also be used in reverse. An example of this is the dispersion of solid polymer rotating spheres, produced by Cairns. The polymer matrix is used to bind the liquid crystal in spherical cavities within the film. When an electric field is applied, the bipolar director field of the droplets will align with the electric field, which allows light to pass through the film. The solid polymer spheres were dispersed in glycerol and rotated when an electric field is applied. In another example of reverse PDLC, the size of the liquid crystal droplets was changed by changing the concentration and cooling rate (before polymerization). ${ }^{[59]}$ This resulted in polymer spheres having a strong adhesion both on glass and plastic surfaces.

Using anopore membranes as a confining template, highly ordered microstructures including, toroids, yarn balls and rods have been produced by Cairns.

Multiple mathematical models that describe the PDLC reaction to an electric field have been developed and will be discussed below. ${ }^{[60-63]}$

PDLC flakes have also been reported. ${ }^{[64]}$ PDLC flakes suspended in a host fluid can be controlled with an AC electric field. The field acts to induce a dipole moment on the flake. The results showed that the flakes would reorient to align one of the two major axes parallel to the electric field. 


\subsubsection{Fabrication Methods}

\subsubsection{Encapsulation}

Some early attempts to produce PDLCs were using the technique of encapsulation. ${ }^{[65]}$ In this technique, liquid crystal mixed with a polymer is dissolved in water and the mixture is left until the water is evaporated. Thus leaving the liquid crystal distributed in a polymer matrix as small capsules. The liquid crystal capsules or droplets tend to not be uniform in size and not distributed evenly throughout the polymer matrix.

\subsubsection{Phase Separation}

When producing PDLCs through phase separation a mixture of liquid crystal and polymer is produced. The liquid crystal droplets are then phase separated from the polymer. This is achieved using two different methods: namely, polymerization induced phase separation and thermally induced phase separation or solvent induced phase separation.

\subsection{Polymerization Induced Phase Separation}

When polymerization induced phase separation takes place, a liquid crystal is mixed with a low molecular weight monomer that has yet to undergo polymerization, acting as a solvent for the liquid crystal. ${ }^{[65]}$ Once the solution is formed, the polymerization is induced through radiation, heat or light. The use of UV light as to polymerize the monomers was discovered early on in the production of PDLCs. As the solution is being polymerized, the polymer chains phase separate from the liquid crystal as the liquid crystal forms droplets. The droplets grow until the polymer becomes solid enough to trap them. The phase separation process is sensitive to temperature and light intensity, which influences the liquid crystal droplet sizes, as well as the type of materials and the amount of materials used. It has been reported that a small change in the composition, temperature or speed of polymerization can greatly 
influence the size of the droplets which can lead to non-uniformity in the PDLC film or difficulty in producing the same film multiple times.

\subsection{Thermally Induced Phase Separation}

When thermally induced phase separation takes place, a thermoplastic polymer is heated into a melt and is mixed with a liquid crystal to form a single-phase solution. The solution is cooled at a specific rate and induces the phase separation. The liquid crystal phase separates into droplets as the solution cools and the droplets grow until the polymer hardens around them. Based on the cooling rate, the size of the droplets will change. When the solution is cooled rapidly, the droplets are smaller compared to a slow cooling of the solution because the solution does not have time for large particles to form. The PDLC films that are produced using this type of phase separation are often sensitive to processing history. These are difficult to replicate and unstable at high temperatures. ${ }^{[65]}$

\subsection{Solvent Induced Phase Separation}

When solvent induced phase separation takes place, the liquid crystal and polymer are dissolved in a solvent, typically organic, to form a single-phase mixture. The solvent is then removed through evaporation at a controlled rate. The liquid crystal phase separates out as droplets within the polymer matrix and stops when all of the solvent has been removed. By controlling the rate that the solvent is removed, the droplet size can be controlled. Similar to thermally induced phase separation, the liquid crystal droplet size increases as the rate at which, the solvent is removed decreases. ${ }^{[65]}$ 


\subsubsection{Varying Concentration}

Based on the required size of liquid crystal droplets within PDLC films, a film can be produced to reflecting the necessary droplet size. By controlling the polymerization and by adjusting the concentrations of the PDLC mixture components, different types of sample morphologies can be obtained. ${ }^{[66-68]}$ In PDLC films compared to well-defined pores of nuclepore and anopore membranes, the size distribution can be large. ${ }^{[68]}$ The size of the liquid crystal droplets within a PDLC film was changed by stretching the film uniaxially $5 \%, 10 \%, 100 \%$ and $400 \%$ of the original length. ${ }^{[58]}$ An ultrasonic processor was used to emulsify the liquid crystal into a PVA aqueous solution. In addition, droplet size can be changed by increasing the concentration of liquid crystal within the PDLC film. ${ }^{\text {[69] }}$ When the concentration of liquid crystal is high, the morphology of the films is determined primarily by the rate of solidification of the polymer matrix. ${ }^{[70]}$

Polyvinyl alcohol (PVA), which is a water-soluble synthetic polymer, has been shown to align the liquid crystal without defects. ${ }^{[71]}$ In addition, at a weight percent concentration of 20, the solubility problems are minimal. ${ }^{[72]}$ When created PDLC films with PVA, the films are typically stretched at a temperature range of $150-200^{\circ} C^{[73,74]}$ and the aqueous PVA solution is allowed to evaporate at a slow rate to increase the tensile strength. ${ }^{[75]}$ 


\section{Chapter 3: Applications}

\subsection{Morphing Wings}

An application of electro-active liquid crystal dispersed composites is morphing wings. With fuel prices increasing, as well as the average consumer's free time decreases, a need for faster and more cost efficient travel is now more important than ever before. Shape-shifting or morphing wings intend to minimize fuel consumption, air resistance or drag, reduce noise and emissions, maximize cruise efficiency as the aircraft burns off fuel and require less lift. Instead of completely replacing the wing or even creating a completely new aircraft, which is the pricey and time-consuming concept of current morphing projects, ${ }^{[75-78]}$ a film can be attached to the surface of the aircraft wing. The major advantage of this application compared to current attempts of morphing wings is the cost efficient design; thus, this project provides both large commercial value and social impact.

\subsection{Active Micromixer}

One of the essential microfluidic operations required is rapid and complete mixing of two or more solute streams. ${ }^{[79]}$ Active mixers that use a variety of phenomena, such as magnetic stirring, ${ }^{[80]}$ electrokinetic instabilities ${ }^{[81]}$ and acoustic micro-streaming actuation ${ }^{[82]}$ have been reported. By using the PDLC film and attaching it to a channel wall, the film can be used as an active micromixer. The rods can be controlled by an external stimulus such as an electric field and thus be used as an active Micromixer. Computational fluid-structure models based on artificial cilia have been demonstrated. The results showed that with a proper actuation scheme, as few as two micro-actuators placed on the same wall of a micro-channel can induce effective mixing by chaotic advection. Chaotic advection describes fluid motion in which particles rapidly and abruptly change trajectories. 


\subsection{Displays}

Another potential application is in a display. Traditional liquid crystal displays (LCDs) use polarizers to allow the transmittance of proper oriented light. ${ }^{[83]}$ However, a display using blackpigmented liquid crystal polymer rods dispersed in a polymer does not need polarizers, and the rods can act as light modulators as shown in figure 3.3-1.

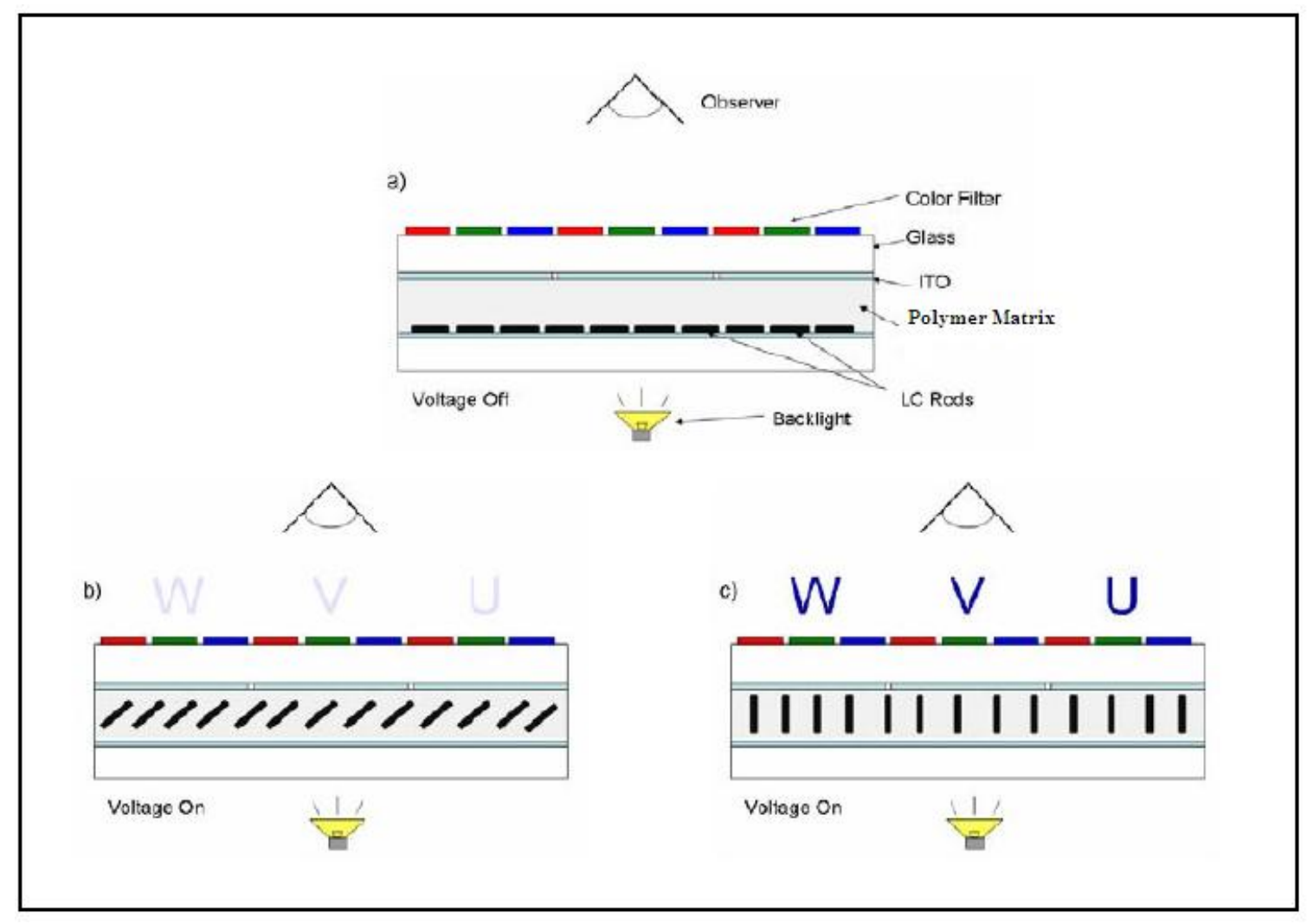

Figure 3.3-1: a) Display OFF, b) Display on MEDIUM, c) Display HIGH ${ }^{[15]}$ 
The main drawbacks of polarizers are their poor efficiency, their degradation over the life span of the device and their high cost to manufacture. The absence of a polarizer would therefore reduce the total cost of the devices as well as the power consumed. In the OFF state Figure 3.3-1a, rods would block light from traveling through the device. In the MEDIUM state Figure 3.3-1b, the rods would slightly align within the polymer matrix and would allow a grayscale to be achieved. In the HIGH state Figure 3.3-1c, the rods would allow the maximum amount of light through the cell. 


\section{Chapter 4: Materials, Equipment and Fabrication}

\subsection{Materials}

\section{Reactive Mesogen:}

The liquid crystal used was RM257 (manufactured by Merck). RM257 polymerizes by UV curing and is a diacrylate reactive liquid crystal. The nematic phase and clearing points are $67^{\circ} \mathrm{C}$ and $127^{\circ} \mathrm{C}$. In its bulk state, it is a white powder. RM257 has the following chemical formula: 4-(3acryloyloxypropyloxy)phenyl 4-[4-(3-acryloyloxypropyloxy)benzoyloxy]-2-methylbenzoate).

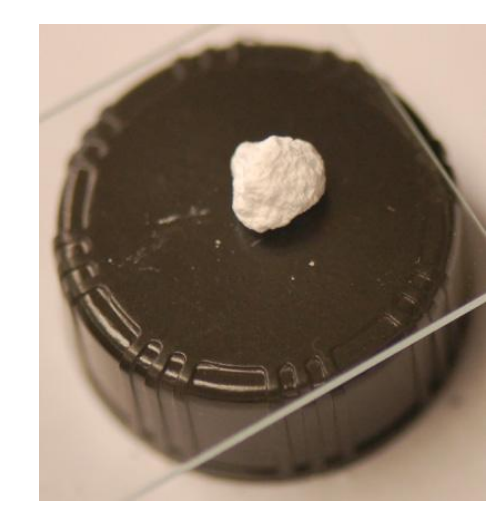

Figure 4.1-1: RM257 in its bulk state

\section{Photo-initiator:}

The photo-initiator is Daracur 1173 (manufactured by Ciba Specialty Chemicals Inc.) and will be mixed with the RM257. A photo-initiator is the catalyst that starts the chain reaction during UV curing. Daracur 1173 has the following chemical formula: 2-hydroxy-2-methyl-1-phenylpropanone. 


\section{Spacers:}

The spacers are spheres $10 \mu m$ in size with a standard deviation of $<2 \mu m$ (coefficient of variance $<2 \%$ ). The spacers are used to separate the ITO glass and are made of polymethacrylate. As packaged, the particles are dispersed in water with a concentration of $10 \%$ solid.

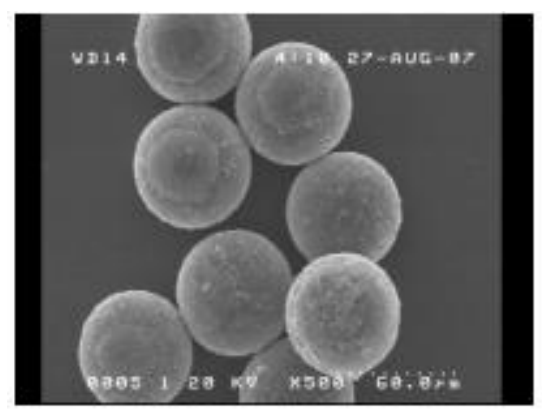

Figure 4.1-2: SEM image of spacers

\section{Anopore Membrane:}

The anopore membrane (manufactured by Whatman, Anodisc 13), is $200 \mathrm{~nm}$ in diameter, $60 \mu \mathrm{m}$ in thickness, and has a porosity of $40 \% .{ }^{[37]}$ An anopore membrane is constructed by the anodic oxidation of aluminum and is used as the confining medium. The membranes are also very brittle and must be handled with care.

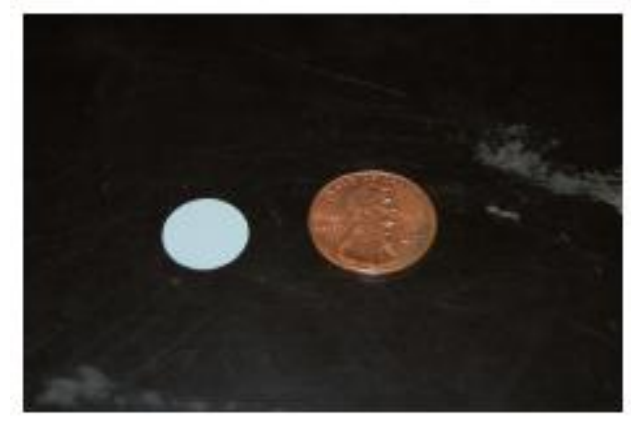

Figure 4.1-3: Anopore membrane with a penny for size comparison 


\section{Dispersion Fluids:}

The silicon oils were chosen because they are completely transparent, they exhibit low volatility and have a wide range of viscosities. By varying the viscosities it is expected that an increase in movement time as well as different rod movements to occur.

\section{Indium tin oxide glass (ITO):}

Indium tin oxide (ITO) is transparent. It was applied to one surface of the glass, causing that surface of the glass to be conductive. The ITO glass is used to make the glass cells.

\section{Optical Microscope:}

The rod movement was observed using an optical microscope (Leitz LaborLux $12 \mathrm{ME}$ ). During analysis, optical images were captured with a frame grabber (Guppy, Allied Vision Technology). 


\subsection{Equipment}

\section{Hot Plate:}

A Corning modified hot plate was used during the preparation of the samples, which is shown in Figure 4.2-1. The Corning hot plate was modified to fit in the curing chamber and to keep the sample away from the heat of the mercury lamp inside the curing chamber. The hot plate was necessary to fit within the curing chamber so the liquid crystal could be within the nemantic phase while curing. The top of the hot plate is constructed from steel with a white enamel finish and has a temperature range of $25-355^{\circ} \mathrm{C}$.

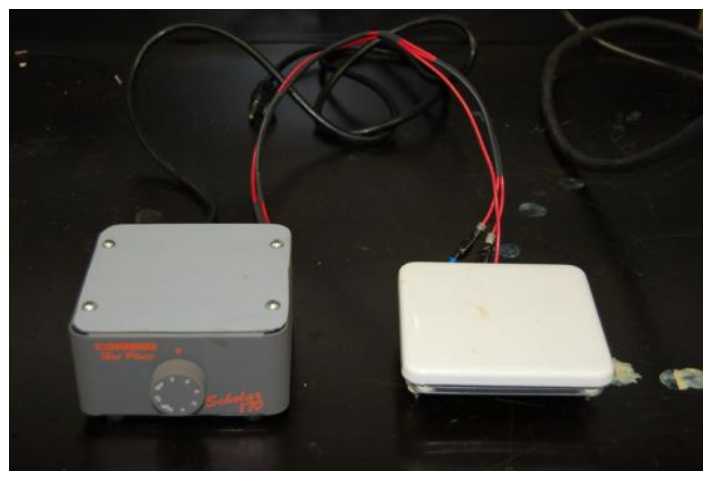

Figure 4.2-1: Corning modified hot plate

\section{Sonicator:}

A sonicator (Branson $1510 \mathrm{R}, 42 \mathrm{kHz}$ ) was used to sonicate the rods. Figure 4.2-2 shows the sonicator used.

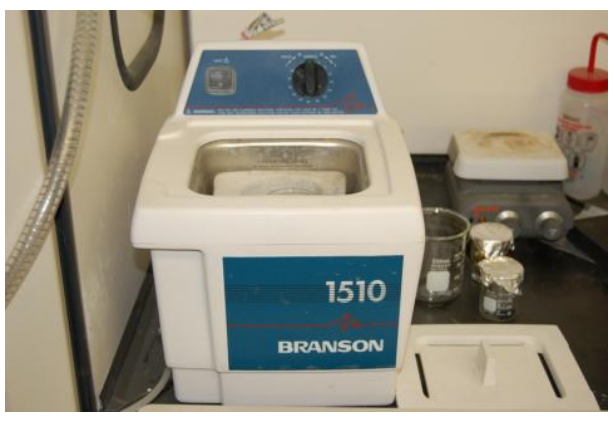

Figure 4.2-2: Branson snicator (42 kHz) 


\section{Curing Chamber:}

The curing chamber and lamp house (manufactured by UVEXS, California) is shown in Figure 4.2-3. The lamp house contains a mercury vapor lamp, which is able to supply UV light in the 200-400 $\mathrm{nm}$ spectrum. The lamp housing is also fitted with a reflector that filters out up to $95 \%$ of infrared spectrum light. This helps to ensure that excess temperature from the lamps is kept to a minimum.

The power supply is separate from the curing chamber and is shown in Figure 4.2-4. The power supply is able to adjust the intensity of the lamp 150 (low), 200 (medium), 300 (high) Watts per inch. In addition, the power supply will automatically time the shutter speed so curing time can be made standardized for the samples.

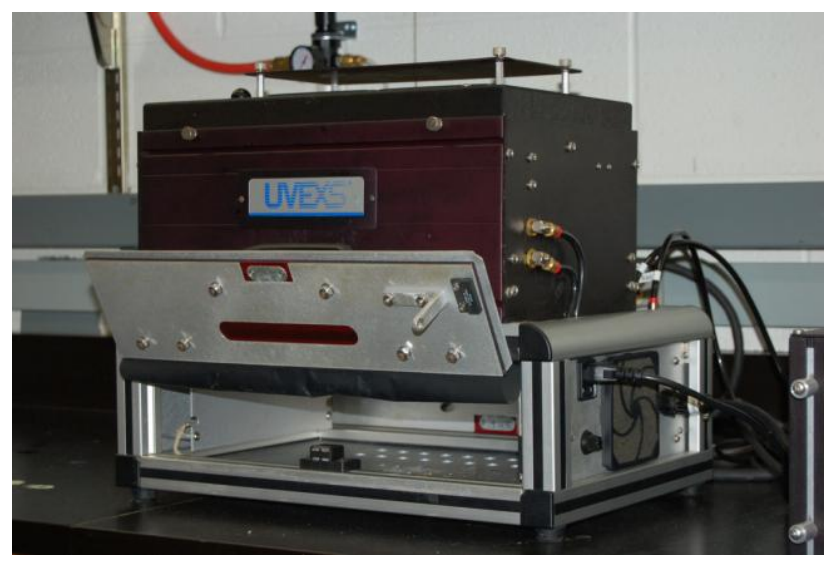

Figure 4.2-3: Sample Chamber (bottom), Lamp House (top)

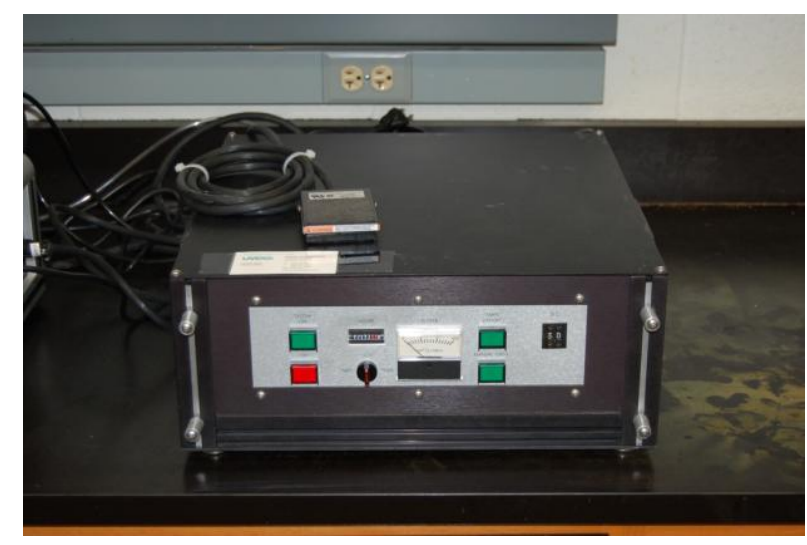

Figure 4.2-4: Power Supply 


\subsection{Fabrication of Rods}

\section{Mixing:}

At a weight percentage of 95\% RM257 and 5\% photo-initiator, the mixture is combined in a glass vial. The mixture is then placed on a hot plate to bring the RM257 to its liquid crystal phase. Once the RM257 has liquefied, the mixture is manually stirred with a pipette to ensure that the photo-initiator and RM257 are mixed thoroughly.

\section{Filling the Anopore Membrane:}

The anopore membranes are broken into smaller pieces to make fabrication more manageable and to reduce the amount of waste. A glass slide is placed on the hot plate and a droplet of RM257/photoinitiator mixture is placed on the glass slide. The anopore membrane is now placed on the edge of the RM257/photo-initiator droplet. The anopore membrane will fill by capillary action. On top of the membrane, some of the extra RM257/photo-initiator will gather causing a thick polymer top, which will lead to problems during sonication. To prevent this problem, the filled membrane is pressed between two filter papers, removing the excess RM257/photo-initiator, but not removing all of the mixture from the membrane.

The filled membrane can now be placed back onto the glass slide and moved to the hot plate that is in the curing chamber. The temperature of the filled membrane is monitored by a thermocouple (ktype). The thermocouple leads are placed between the glass slide and the hot plate. The thermocouple is held in place by weights placed on top of the glass slide and had a resolution of $\pm .05^{\circ} C$. A temperature rise of $1-4^{\circ} C$ was noted when on the low power setting of 150 per inch. An image of this set-up can be seen below. 


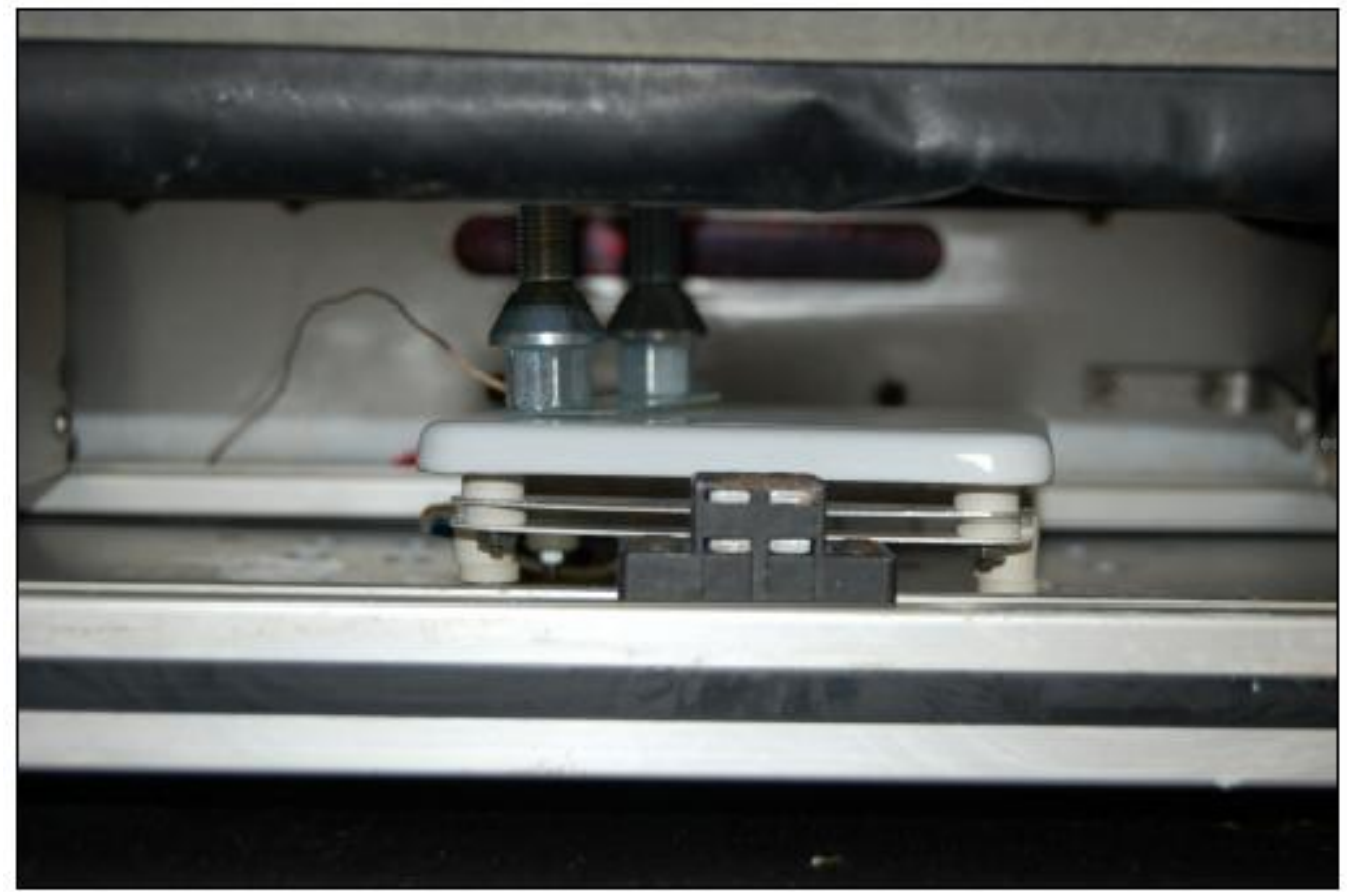

Figure 4.3-1: Thermocouple monitoring temperature of hot plate with weighted glass slide on top

\section{Curing:}

The filled membrane is placed on the hot plate inside the curing chamber and then the curing chamber is set for the prescribed temperature and time. The curing chamber was set on the low power setting, 150 per inch, for 30 seconds. These settings where chosen based on past work completed by Shafran 2008. After curing for 30 seconds on the low power setting, the liquid crystals polymerized into solid polymer rods since they were confined in the anopore membrane.

\section{Etching:}

Separating the rods from the membrane is achieved by etching the membrane, leaving only polymerized liquid crystal cylinders. Using a $0.04-0.06 \mathrm{M}$ solution of $\mathrm{NaOH}$, the membrane was place in a vial containing the solution. The $\mathrm{NaOH}$ solution will react with only the anopore membrane. Etching will take approximately 2 hours to complete and is noted by the membrane becoming flexible. 


\section{Sonication:}

Once the membrane has been etched, the $\mathrm{NaOH}$ solution is removed and the film is washed with distilled water. Once this is complete, ethanol is added to the glass vial and is placed in a sonicator for 18 minutes. Other times where attempted for sonicating the rods however, they appeared better dispersion of the rods where observed at 18 minutes. This leaves the polymer rods dispersed in ethanol. Finally, the ethanol is allowed to evaporate at room temperature, for 24 hours, leaving only rods.

After the ethanol has fully evaporated, low, medium, or high viscosity liquids are added to the vial of rods. The mixture is once again sonicated for 18 minutes to disperse the rods in the mixture completely. The complete procedure is outlined in Figure 4.3-2.

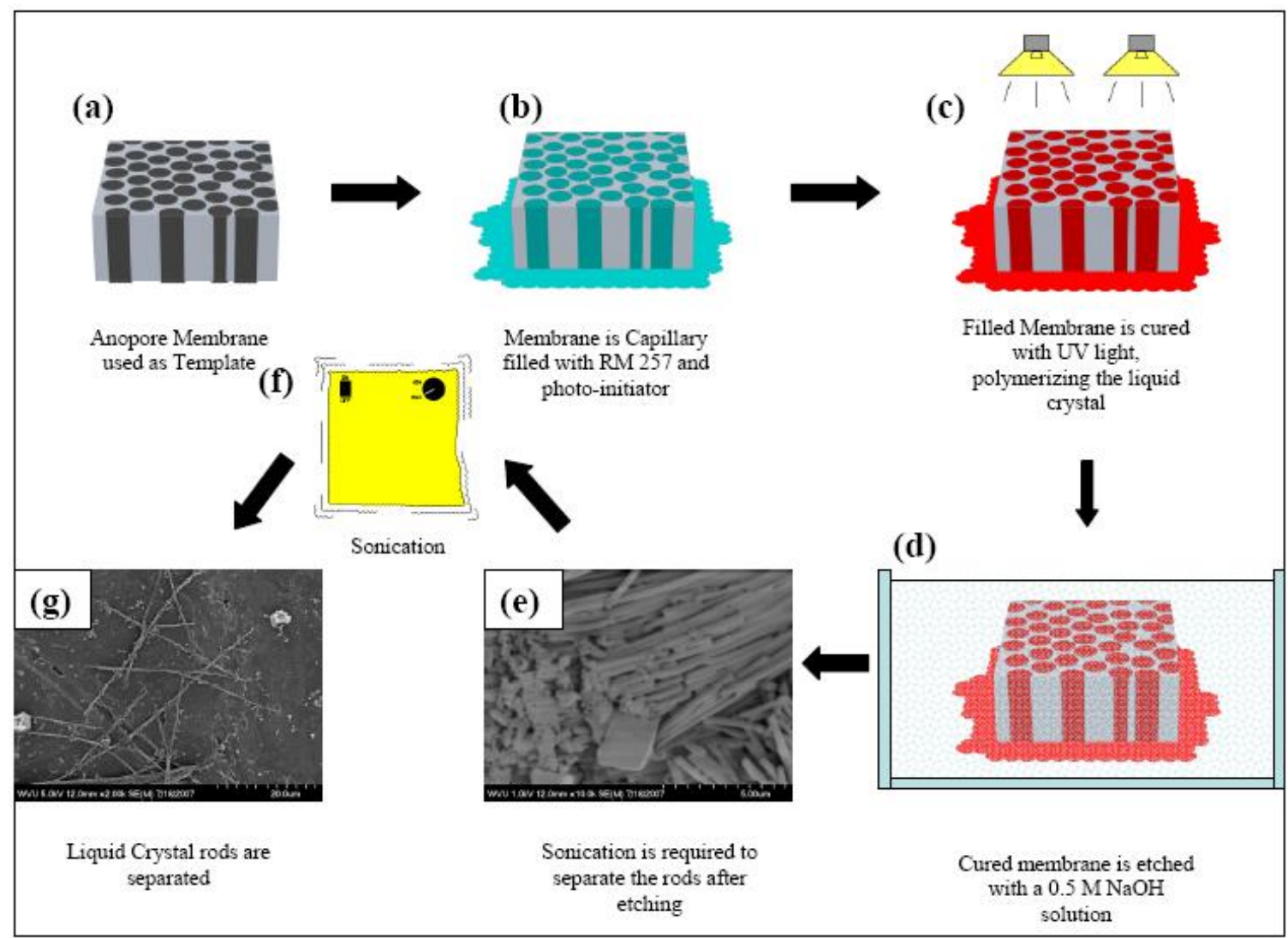

Figure 4.3-2: Fabrication of rods outline ${ }^{[15]}$ 


\subsection{Fabrication of Glass Cells}

The glass cells are fabricated using 1.0 X 1.0 inch piece of ITO glass. The ITO sides of the glass pieces are thoroughly cleaned with acetone and a lint free cloth to remove as much residue and dust particles as possible. Tacky adhesive is also used to remove any particles left behind from the acetone and cloth. The ITO sides of the glass pieces are cleaned thoroughly using acetone and tacky adhesive to ensure that the bottom and top ITO surfaces do not short circuit, rendering the glass cell inoperable.

The top and bottom glass pieces are separated by using polymethacrylate (PMA) spheres. The spheres create a $10 \mu \mathrm{m}$ gap between the two surfaces. The size of the spheres was chosen because the maximum rod length is $60 \mu \mathrm{m}$ and will allow for full rotation of the rods within the glass cell. The PMA sphere and water solution is diluted in ethanol to allow the solution to evaporate fast. The solution is mixed with a weight percentage of $95 \%$ ethanol and $5 \%$ sphere/water mixture.

A clean piece of ITO glass is placed ITO face up and a droplet of the sphere/water/ethanol mixture is placed on it. After 5-10 minutes, the ethanol will be completely evaporated, leaving only spheres on the ITO surface. Once this is complete, another piece of ITO glass, ITO side down, is placed on top of the first piece of ITO glass. The ITO glass pieces should be staggered to allow the attachment of leads. Epoxy glue is now used to glue two sides of the glass cell.

Once the glue has dried, the rod mixture can now be added to the glass cell. The glass cell is filled from capillary action. Once the cell is completely filled, the remaining sides of the glass cell are glued with epoxy. After the epoxy has dried, two leads are attached to the glass cell with conductive silver paint. The complete procedure is outlined in Figure 4.4-1 and a typical glass cell can be seen in Figure 4.4-2. 


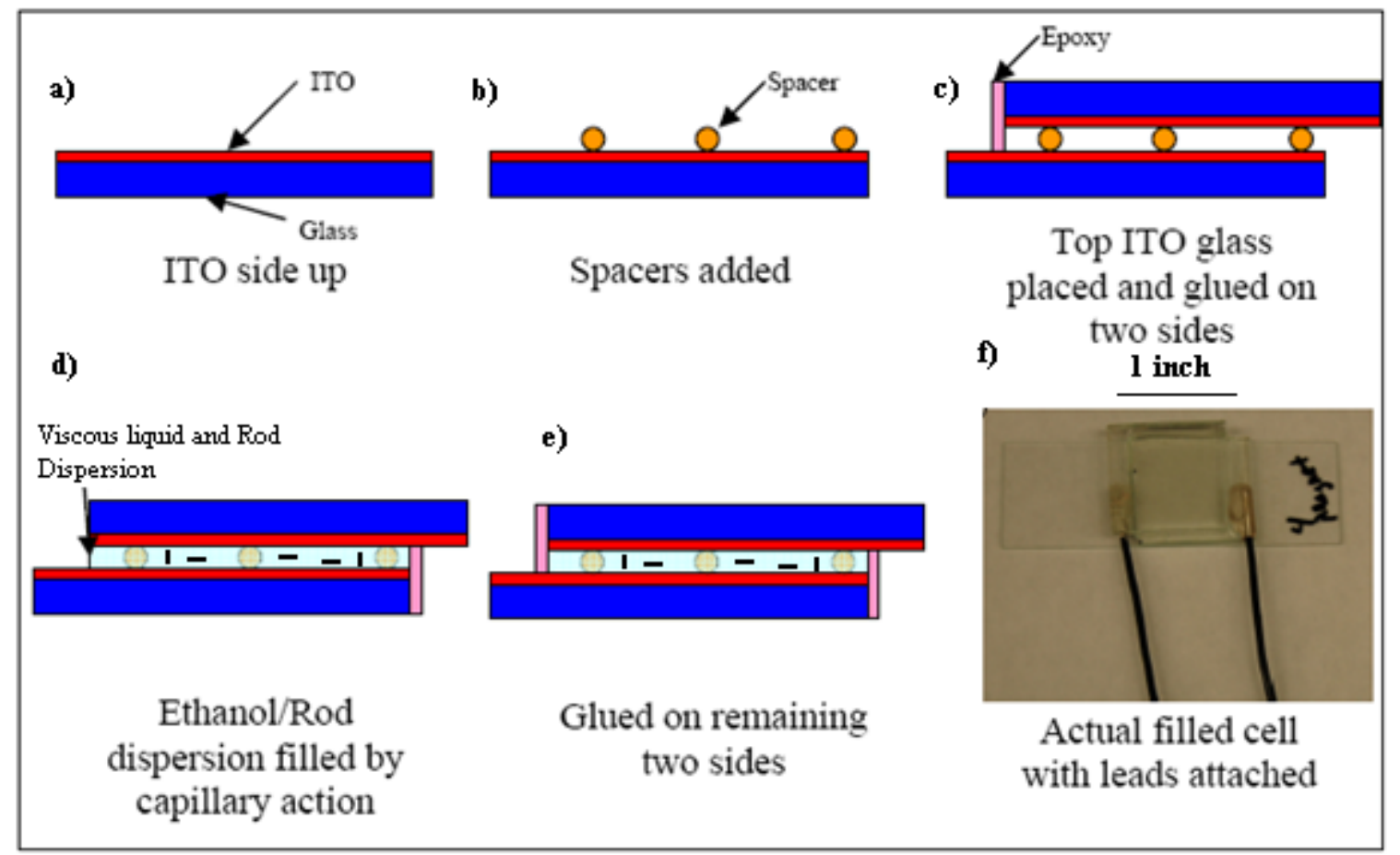

Figure 4.4-1: Fabrication of glass cell outline ${ }^{[15]}$

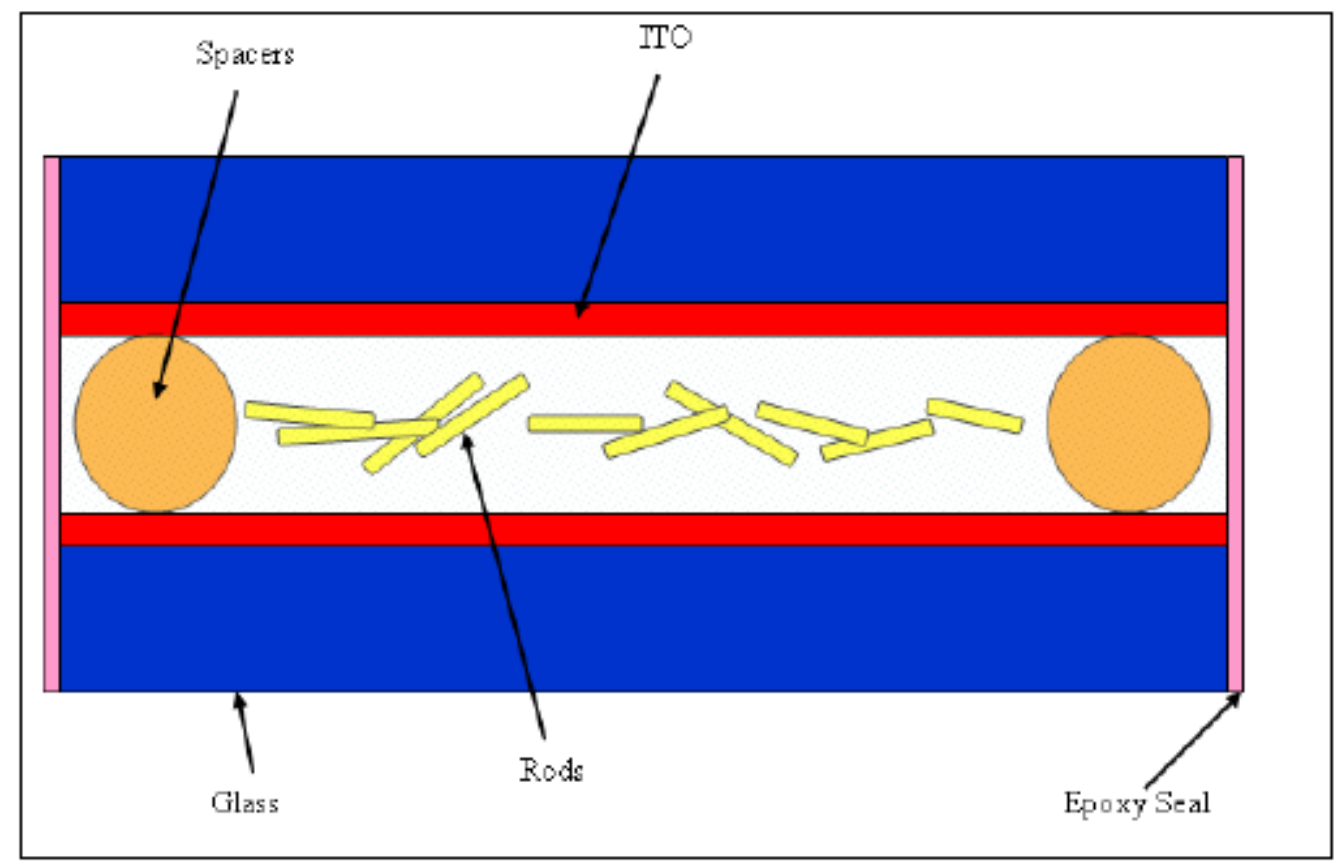

Figure 4.4-2: Side view of typical glass cell ${ }^{[15]}$ 


\section{Chapter 5: Results}

\subsection{Fabrication}

Successful repeatable fabrication of the liquid crystal rods had already been accomplished from Shafran. ${ }^{[15]}$ Some minor problems including polymer top thickness which doesn't allow the rods to separate, and improper curing temperature from opening and closing the curing chamber, created uncured rods.

One of the major problems encountered during the fabrication process was dispersing the rods within the fluid. Since the viscosity of the dispersion fluids was large, the rods had trouble moving freely through the fluid during sonication. To rectify this problem, when the rods were mixed with the dispersion fluid the sonication time was increased for 18 minutes to 45 minutes for all dispersion fluid cases.

The glass cells, since filled by capillary action, were slow to fill, especially as the viscosity of dispersion fluid increased. To help with this problem, the glass cells where tilted during filling.

\subsection{Response to Applied Electric Field}

The rods were observed to move with an externally applied alternation current (AC) electric field of 90 . The power supply was connected to the glass cells via the attached wire leads.

With an applied AC field the rods demonstrated many types of movement. Those include spinning about a central axis, large circular motion, and flexing about the center or wagging. 


\subsubsection{Low Viscosity Dispersion Fluid}

When the rods are dispersed in a fluid with a viscosity of $4.7 \mathrm{cP}$, the rod motions that were observed are either spinning about a central point or large circular motions. An ideal representation is shown in Figure 5.2.1-1. During analysis, optical images were captured every $0.1 \mathrm{~s}$. These motions can been see in Figure 5.2.1-2 and 5.2.1-3, respectively.

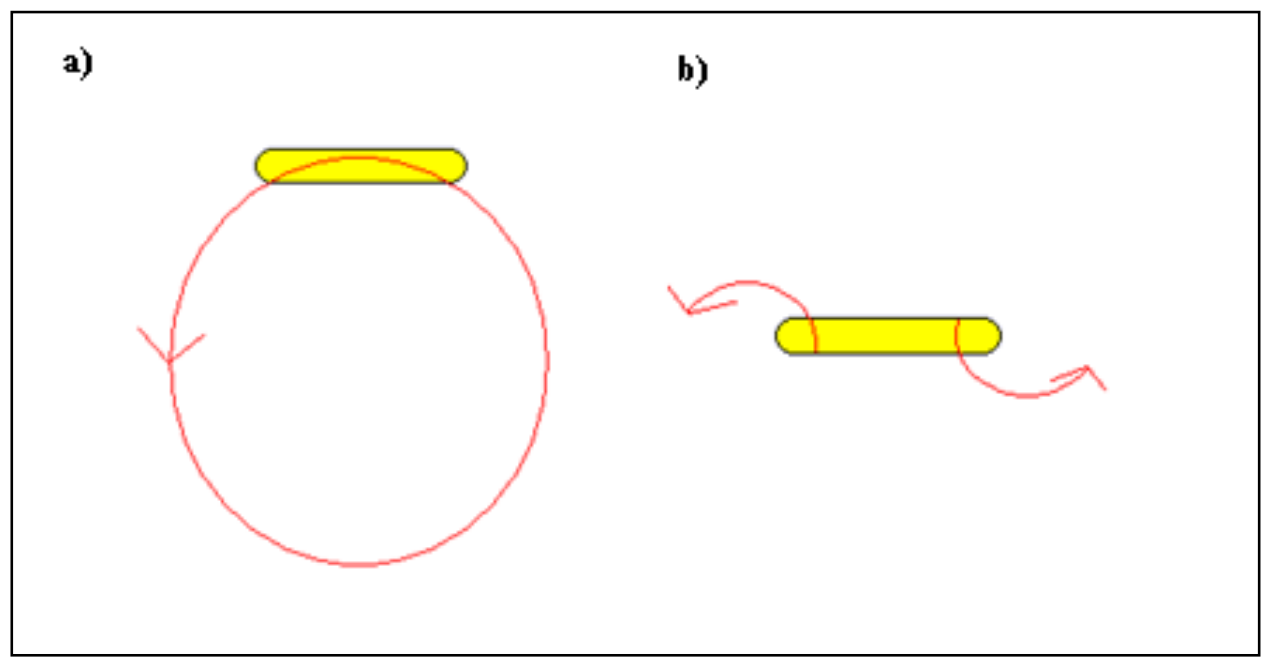

Figure 5.2.1-1: a) rod moving in a large circle (top view) b) Spinning about a central point (top view) 


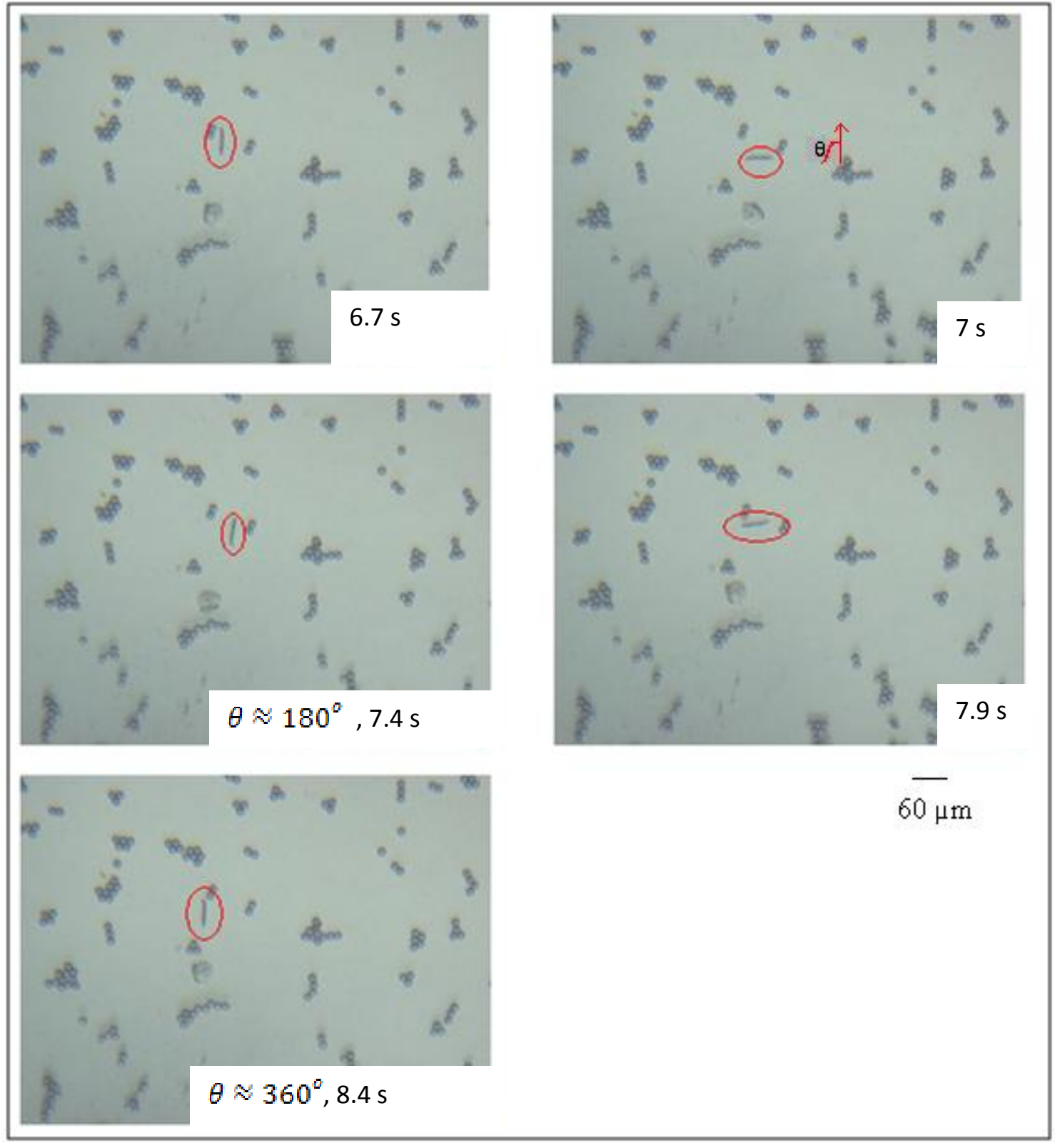

Figure 5.2.1-2: Rod spinning about a central point 


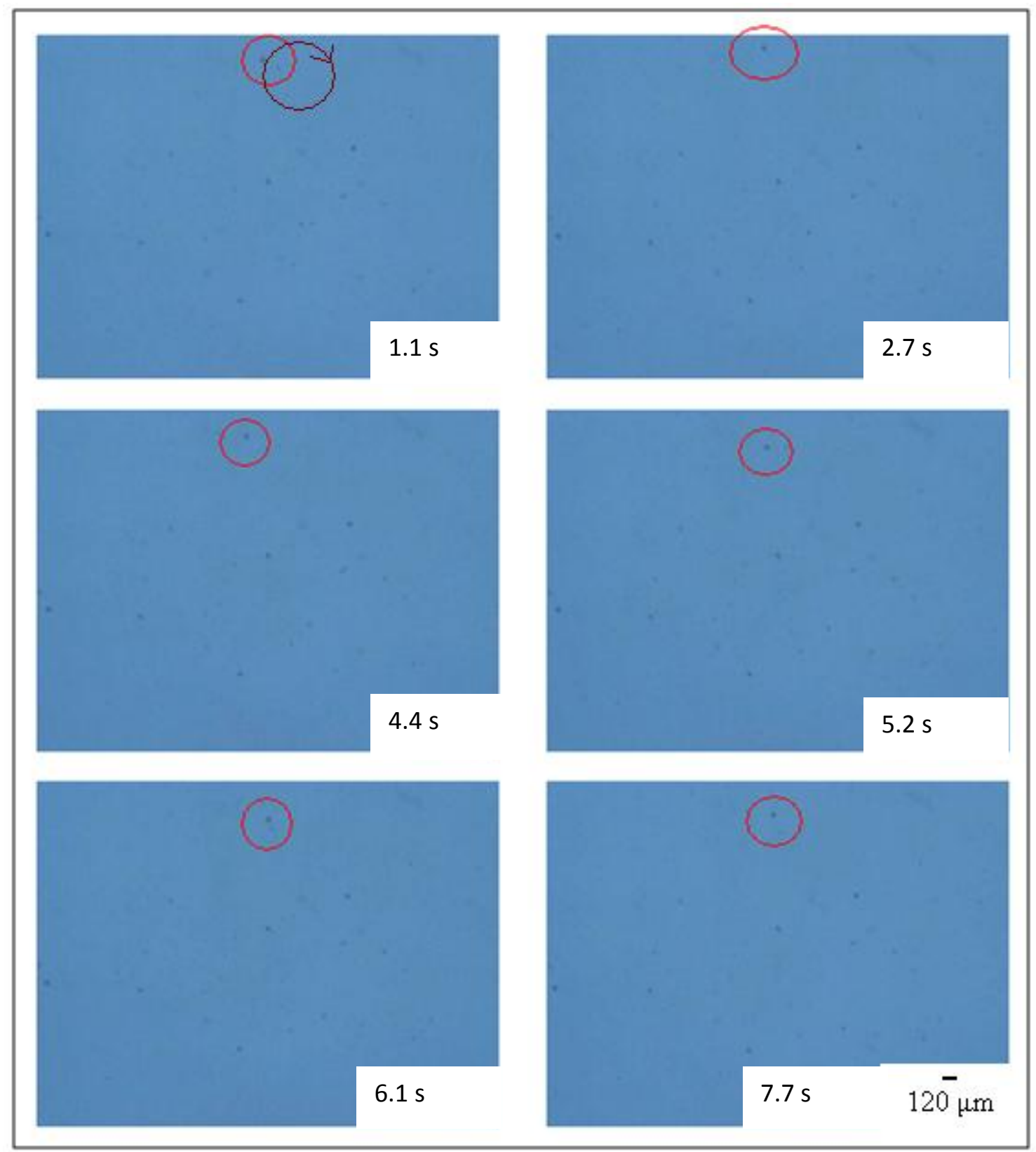

Figure 5.2.1-3: Rod moving in large cricle, where dark red circle shows the approximate path the rod follows during movement 


\subsubsection{Medium Viscosity Dispersion Fluid}

When the rods are dispersed in a fluid with a viscosity of $9.2 \mathrm{cP}$, the motions that were observed are either wagging or flexing. An ideal representation is shown in Figure 5.2.2-1. Two examples of wagging can be seen in Figure 5.2.2-2 and 5.2.2-3 and a flexing rod example can be seen in Figure 5.2.24. During analysis, optical images were captured every $0.55 \mathrm{~s}$. Frame capture time changes from before because the time required for the rods to move increased.

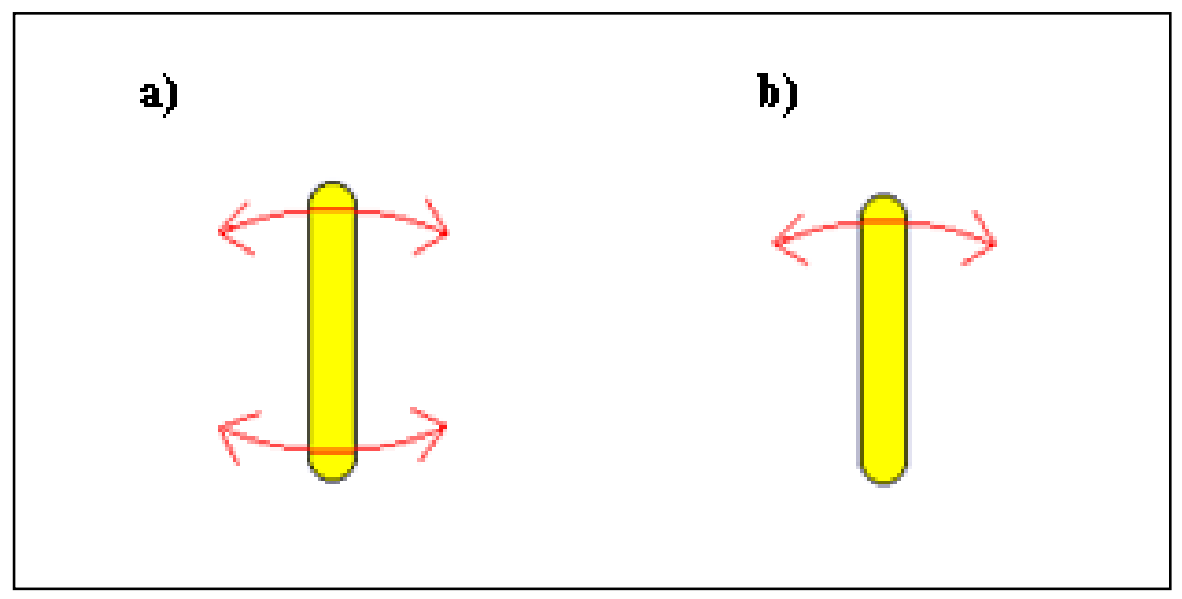

Figure 5.2.2-1: a) Rod flexing (top view) b) Rod wagging (top view) 


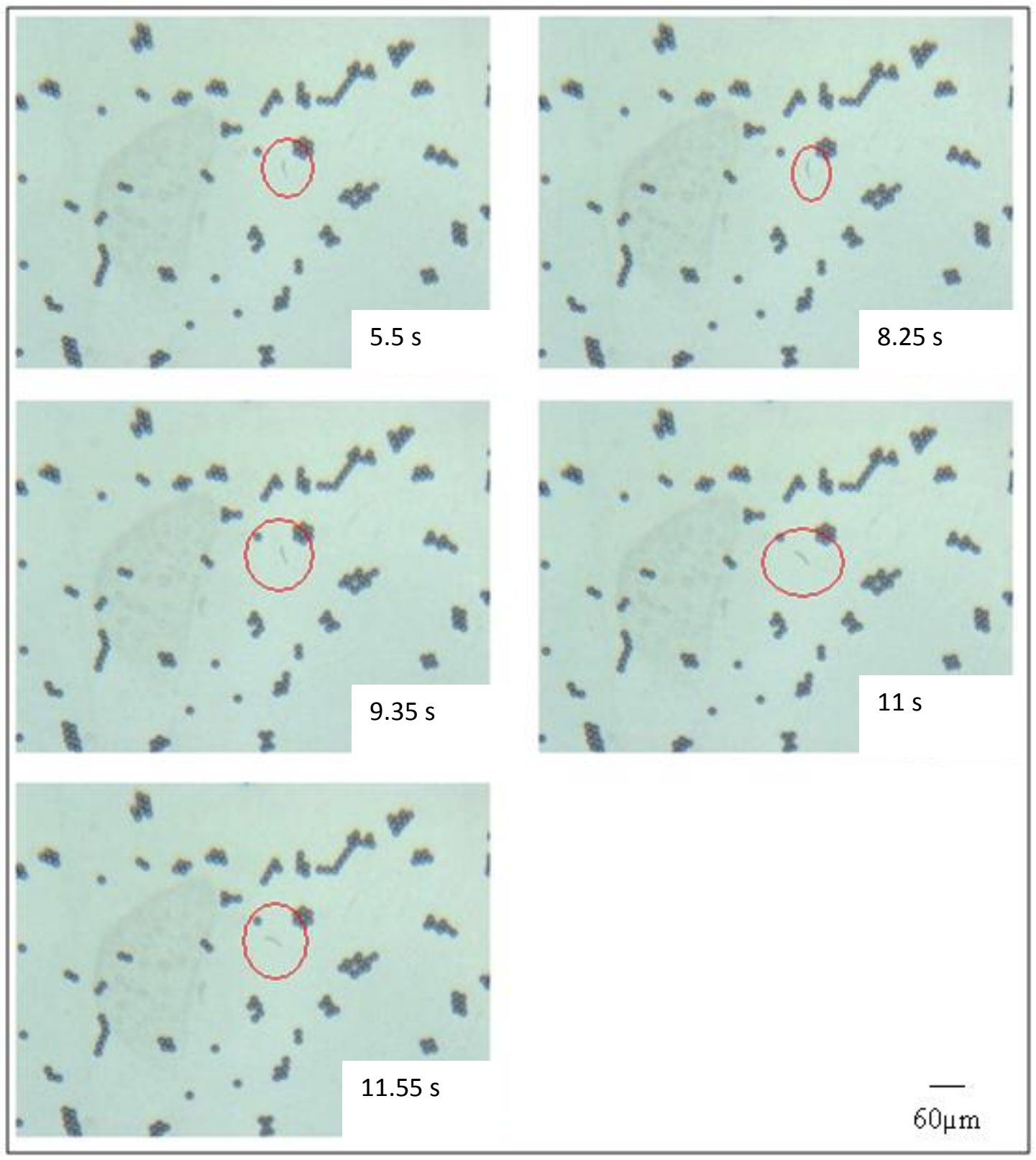

Figure 5.2.2-2: Rod wagging 


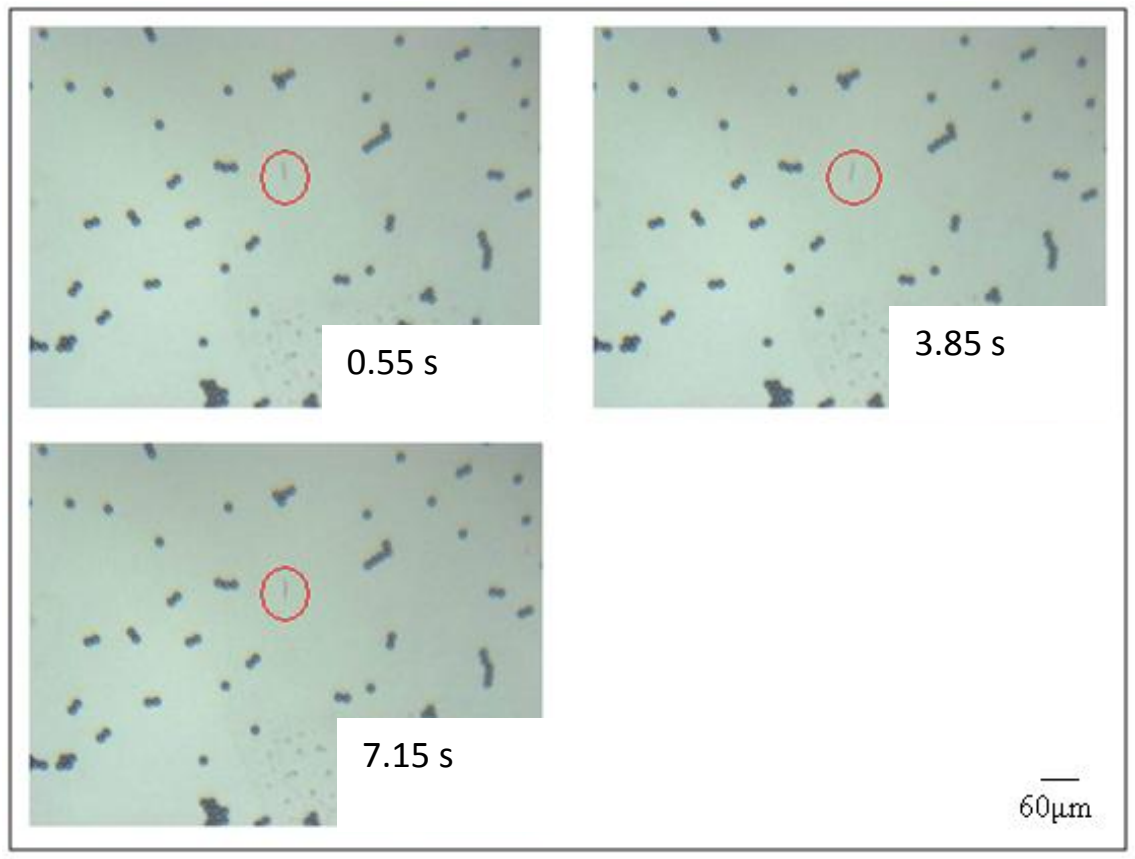

Figure 5.2.2-3: Rod wagging

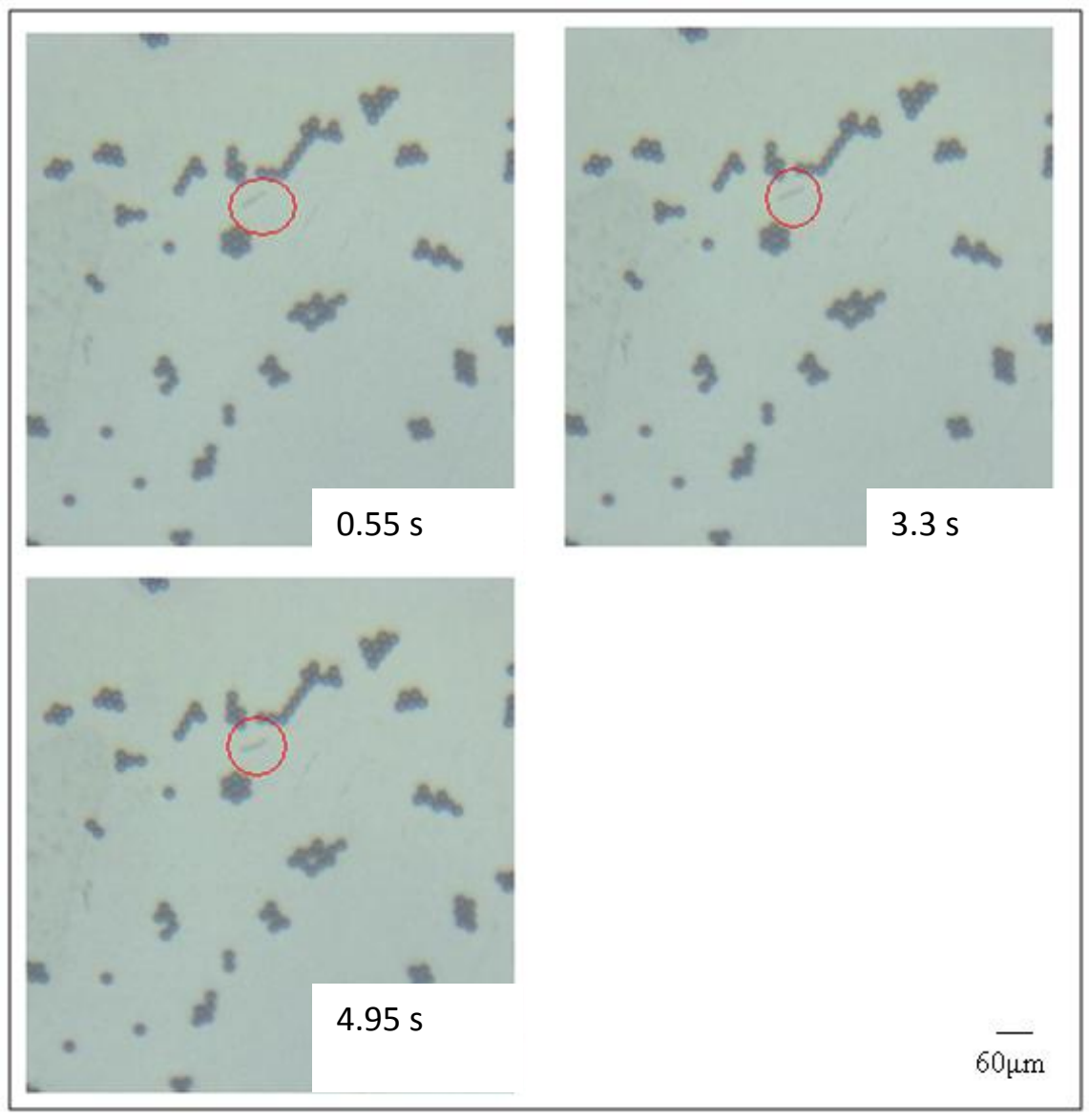

Figure 5.2.2-4: Rod flexing 


\subsubsection{High Viscosity Dispersion Fluid}

When the rods are dispersed in a fluid with a viscosity of $99 \mathrm{cP}$, the motions that are observed are large circular motion and wagging. During analysis, optical images were captured every $0.55 \mathrm{~s}$. An ideal representation is shown in Figure 5.2.3-1. This can be seen in Figure 5.2.3-1 and Figure 5.2.3-2.

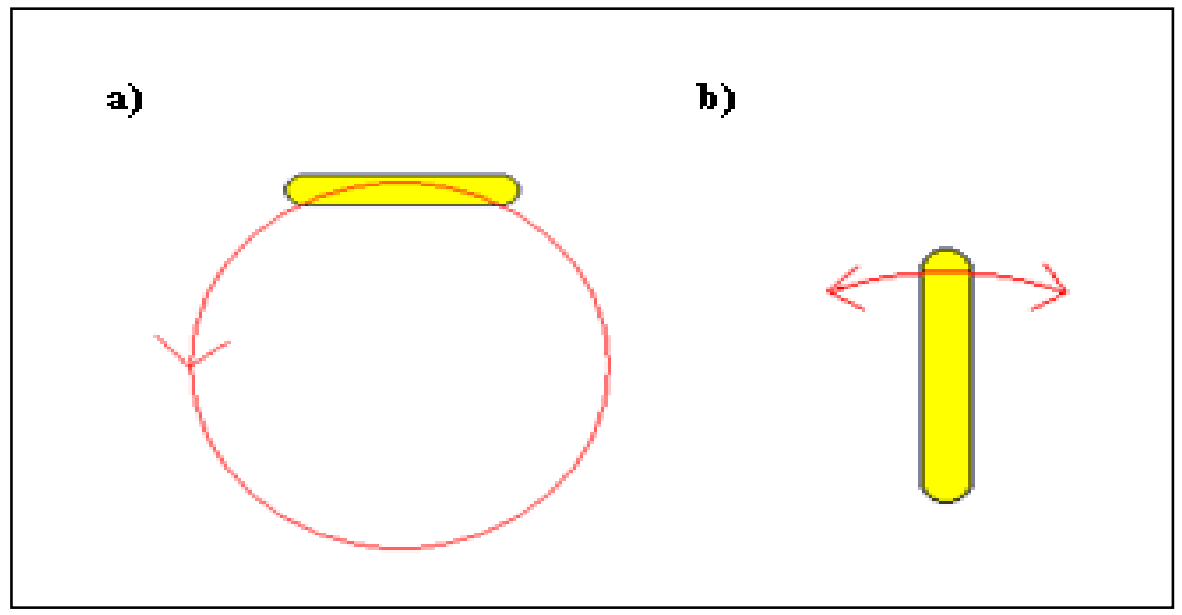

Figure 5.2.3-1: a) rod moving in a large circle (top view) b) Rod wagging (top view) 


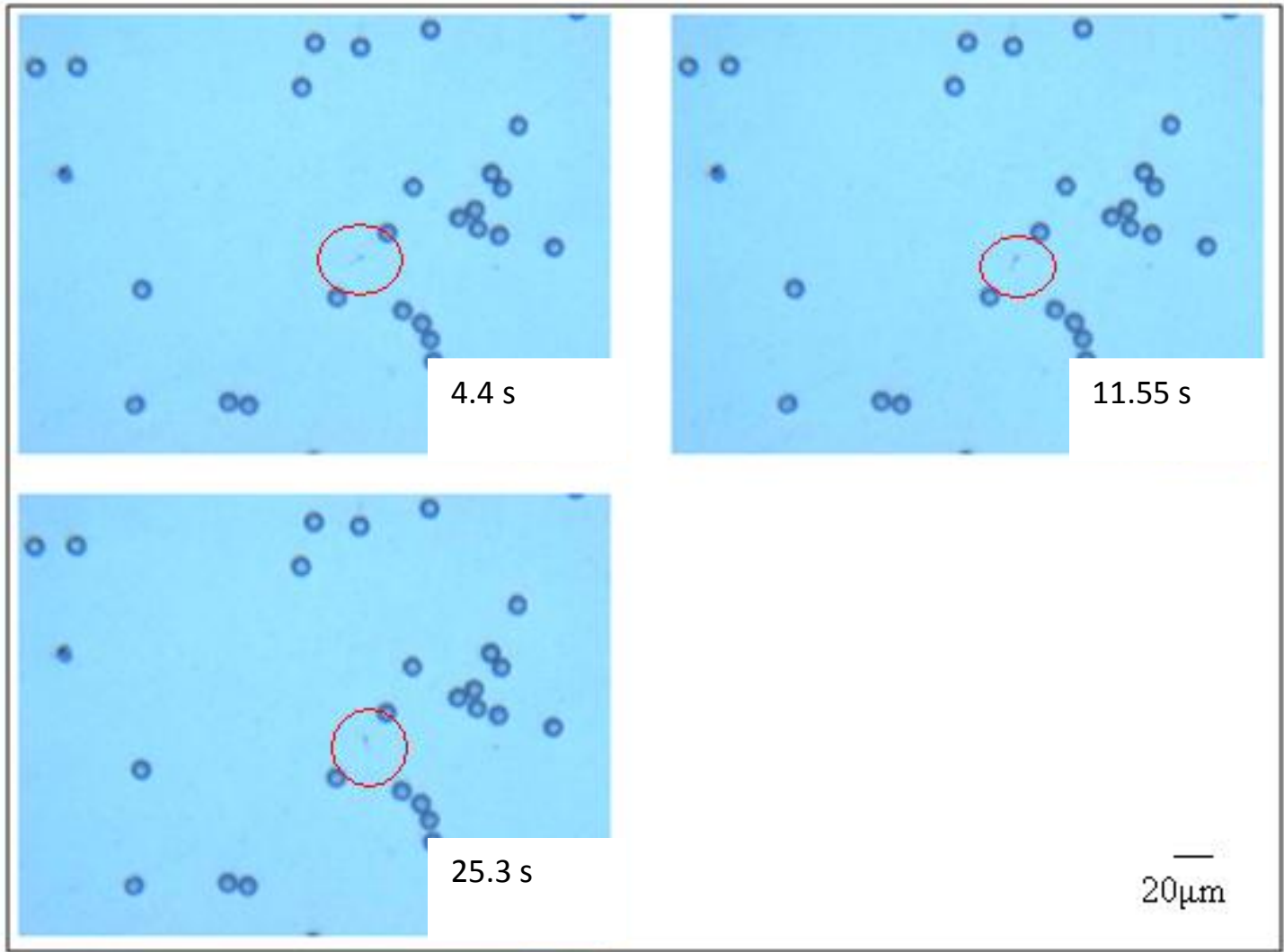

Figure 5.2.3-2: Rod wagging 


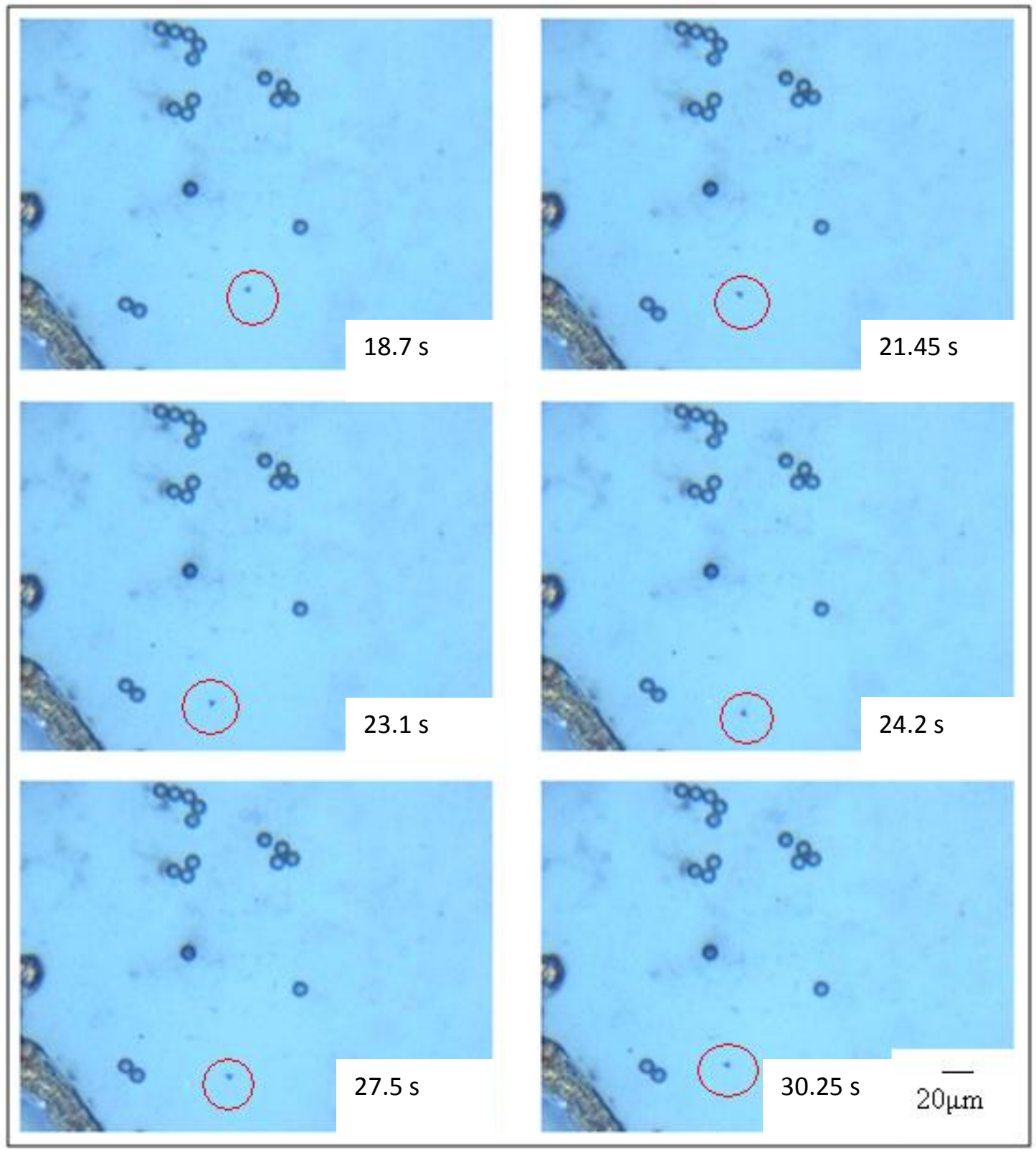

Figure 5.2.3-3: Rod moving in a large circle 


\section{Chapter 6: Discussion}

Liquid crystals can have either a negative or positive dielectric anisotropy value. A negative dielectric anisotropy value will align perpendicular with the applied field. While a positive dielectric anisotropy value with align parallel. RMS257 has a negative dielectric anisotropy value which causes perpendicular alignment with an applied field. The observed movements in the AC field are special cases and are further investigated because the movement is not perpendicular with the ITO plane but rather parallel to it.

\subsection{Movement}

The rods were shown to have movement in low, medium and high viscous dispersion fluids. The polymer rods demonstrated a wide variety of movements. In the silicon oil of viscosity $4.7 \mathrm{cP}$, the rod motions observed are spinning about a central point or large circular movements. In silicon oil of viscosity $9.2 \mathrm{cP}$, the motions observed are either wagging or flexing of the rods. Also, in the silicon oil of viscosity $99 \mathrm{cP}$, the motions observed are large circular movements and wagging.

One explanation of why the rods wag or flex could be because one end of the rod is larger, heavier, or attached to another rod which slows one end down. One end of the rod may be heavier because of liquid crystal excess when filling the membrane. It is difficult to view, because of the limitations on the resolution of the optical microscope, if a rod is attached to another rod. Also since a small defect in the rod can cause a change in behavior, this coupled with the increase in viscosity can be the cause of this type of movement of the rods. Also, an explanation of why the rods moved in large circles could be because multiple rods were attached together. This could be caused by an induced or permanent dipole within individual rods. The 
induced dipole within one rod could attract another rod, causing the two rods to cluster. However, since no clustering was observed, this is not a likely cause.

Finally, since the applied AC field was used at such a large voltage, it is also possible that the ITO was shorted out during the testing of the cells. This would cause diminished efficiency of the cell and eventually result in an inactive cell.

The movements could be attributed to a combination of director fields. It was noticed that none of the rods returned to their original position when the field was turned off. This may be attributed to the residual charging on the ITO surface but at this time it cannot be fully explained.

\subsection{Initial Movement and Viscosity}

No relationship between the time a rod initially moved and the dispersion fluid viscosity was established. Since only a small portion of the whole cell could be viewed at once, it is difficult to determine the initial time at which any of the rods moved. An area of rods was identified first and then a field was applied, but where some of the rods would not move, observation on that area was stopped and the process was restarted in a new area. This occurred in all viscosities of dispersion fluids tested. As well as shorting out of leads and ITO. Since such a high voltage was used, multiple runs on a single cell were difficult which created problems when trying to establish a relationship between initial movement and viscosity. A total of 4 runs for a single cell were the maximum and 5 cells for each viscosity was prepared. Also, an increase in sonication time to 45 minutes from 18 minutes could be another cause for the rods lack of movement. Increased sonication time could have led to fractures within the rods, causing 
the rods to have no reaction when the external field was applied. Finally any irregularity in the cell or the spacers can cause sticking or trapping of the polymer rods, resulting in no movement.

Improper aligned domains in the rods would influence the uniformity of the field director. With the liquid crystal molecules improperly aligned, the director field within the rod would not be uniform resulting in rods with either chaotic motion or no motion. To improve alignment better understand of rod fabrication must be established.

\section{Chapter 7: Conclusions}

This research has shown multiple rod movements within different viscosities of dispersion fluids. The polymer rods produced have been manipulated with an AC field, where the dispersion fluid viscosity greatly affected the rod behavior. Movements have been observed in 4.7, 9.2, and $99 \mathrm{cP}$ viscosities. Movement was observed for all viscosities at 90 Volts.

The movements found were spinning about a central point, large circular movements, wagging, and flexing of the rods. With a powerful DC power supply movement and voltage in the different viscosities could be determined. The AC field lead to chaotic movements which made observations and experiments difficult.

\subsection{Future Work}

Future efforts should focus on the use of surface treatments to control alignment to allow better predictions of rod movement, therefore creating cells for more specific use. Also by known the rod movement, observation would greatly improve.

Another research area should be higher viscosity of dispersion and then the dispersion of liquid crystals in a polymer matrix. Polymer dispersed liquid crystals (PDLC) films can be used for multiple 
applications. One of the largest applications for PDLC's is privacy windows. By changing the orientation of the liquid crystal molecules with an electric field, the intensity of transmitted light is varied. 


\section{References}

1. Nisisako, T., Torii, T., Takahashi, T., and Takizawa Y., "Synthesis of Monodisperse Bicolored Janus Particles with Electrical Anisptropy Using a Microfluidic Co-Flow System.” Advanced Materials. Vol. 18, No.9, 2006. pp. 1152-1156.

2. Barbero, E. J. Introduction to Composite Materials Design. New York: Taylor \& Francis, 1999. pp. 61-68.

3. Fernandez-Nieves, A. "Engineering colloids with optical and geometrical anisotropies: decoupling size monodispersity and particle properties." Soft Matter. Vol. 78, No.2, 2006. pp. 105108.

4. Fernandez-Nieves, A., Cristobal, G., Garces-Chavez, V., Spalding G. C., Dholakia, K., and Weitz, D. A., "Optically Anisotropic Colloids of Controllable Shape." Advanced Materials. Vol. 17, No. 6, 2005. pp. 680-684.

5. Leyva-Diaz, P., Perez, E., and Arauz-Lara, J. L., "Dynamic light scattering by optically anisotropic colloidal particles in polyacrylamide gels." Journal of Chemical Physics. Vol. 121, No. 18, 2004. pp. 9103-9110.

6. Kim, J. W., Larsen, R. J., and Weitz, D. A., "Uniform Non-spherical Colloidal Particles with Tunable Shapes.” Advanced Materials. Vol. 19, No. 15, 2007. pp 2005-2009.

7. Paunov, V. N., and Cayre, O. J., "Supraparticles and "Janus" Particles Fabricated by Replication of Particle Monolayers at Liquid Surfaces Using a Gel Trapping Technique." Advanced Materials. Vol. 16, No. 9-10, 2004. pp.788-791.

8. Shepherd, R. F., Conrad, J. C., Rhodes, S. K., Link, D. R., Marquez, M., Weitz, D. A., and Lewis, J. A., "Microfluidic Assembly of Homogeneous and Janus Colloid-Filled Hydrogel Granules." Langmuir. Vol. 22, No. 21, 2006. pp. 8618-8622.

9. Cairns, D. R., Sibulkin, M., and Crawford, G. P., "Switching dynamics of suspended mesogenic polymer microspheres.” Applied Physics Letters. Vol. 78, No. 18, 2001. pp. 2643-2645.

10. Cairns, D. R., Eichenlaub, N. S., and Crawford, G. P., "Ordered Polymer Microstructures Synthesized from Dispersions of Liquid Crystal Mesogens.” Molecular Crystal and Liquid Crystals. Vol. 352, No. 1, 2000. pp. 275-282. 
11. Hanley, I. W., Introduction to Soft Matter. New York: John Wiley \& Sons, LTD., 2000. pp. 267, 312.

12. Strobl, G., Condensed Matter Physics. New York: Springer, 2004. pp 28.

13. Jones, R. A. L., Soft Condensed Matter. Oxford: Oxford University Press Inc., 2002. pp.104-115.

14. Omar, M. A., Elementary Solid State Physics. Massachusetts: Adison-Wesley Publishing Company Inc., 1993. Ch. 12.

15. Shafran, M. S., Responsive Liquid Crystal Polymer Rods. West Virginia: Thesis, 2008.

16. Sigma-Aldrich, Accelerating Customer's Success through Leadership in Life Science, High Technology and Service. 2009. <http://www.sigmaaldrich.com/sigma-aldrich/home.html>

17. Thiem, H., Strohriegl, P., Shkunov, M., and McCulloch, I., "Photopolymerization of Reactive Mesogen.” Macromolecular Chemistry and Physics. Vol. 206, No. 21, $2005 . \quad$ pp. 2153-2159.

18. Broer, D. J., in Liquid Crystals in Complex Geometries: Formed by polymer and porous networks. (Eds: G. P. Crawford, S. Žumer), Taylor and Francis, London, 1996. Ch. 10.

19. Broer, D. J., Boven, J., and Mol, G. N., "In-situ photopolymerization of oriented liquid-crystalline acrylates." Die Makromolekulare Chemie. Vol. 190, No. 9, 1989. pp. 2255-2268.

20. Collings, P. J., Liquid Crystals: Nature's Delicate Phase of Matter. $2^{\text {nd }}$ Edition. Princeton and Oxford: Princeton University Press, 2000. pp. 4-7, 32-34, 90.

21. Ghent University, Liquid Crystals \& Photonics Group. Belgium: Department of Electronics and Information Systems, 2009.

22. Das, S. K., and Rey, A. D., "Magnetic Field-Induced Shape Transitions in Multiphase PolymerLiquid Crystal Blends." Macromolecular Theory Simulation., Vol. 15, No. 6, 2006. pp. 469-486.

23. Hudson, S. D., and Thomas, E. L., "Frank Elastic-Constant Anisotropy Measured from Transmission-Electron Microscope Images of Declinations." Physical Review Letters. Vol. 62, No. 17, 1989. pp. 1993-1996. 
24. Drzaic, P. S., Liquid Crystal Dispersions. New Jersey: World Scientific, 1995. pp. 1-3, 99.

25. Yin, Y., Shiyanovskii, S. V., and Lavrentovich, O. D., Thermotropic Liquid Crystals. Netherlands: Springer, 2007. pp. 277-295.

26. Barberi, R., Donvent, J. J., Giocondo, M., and Lovane, M., "Bistable nematic azimuthal alignment induced by anchoring competition." Journal of Applied Physics. Vol. 84, No. 3, 1998. id.1321.

27. Hochbaum, A., and Labes, M. M., "Alignment and texture of thin liquid crystal films on solid substrates." Journal of Applied Physics. Vol. 53, No 4, 1982. id.2998.

28. Jain, S. C., and Kitzerow, H. S., "Bulk-induced alignment of nematic liquid crystals by photopolymerization.” Applied Physics Letters. Vol. 64, No. 22, 1994. id. 2946.

29. Crawford, G. P., and Zumer, S., in Liquid Crystals in Complex Geometries: Formed by polymer and porous networks. Taylor \& Francis, 1996. Ch. 1.

30. Amimori, I., Priezjev, N. V., Pelcovits, R. A., and Crawford, G. P., “Optomechanical properties of stretched polymer dispersed liquid crystal films for scattering polarizer applications." Journal of Applied Physics. Vol. 93, No.6, 2003. pp.3248-3252.

31. Crawford, G. P., Ondris-Crawford, R., Zumer, S., and Doane, J. W., "Anchoring and Orientation Wetting Transitions of Confined Liquid Crystals." Physical Review Letters. Vol. 70, No. 12, 1993. pp. 1838-1841.

32. Crawford, G. P., Stannarius, R., and Doane, J. W., "Surface-induced orientational order in the isotropic phase of a liquid-crystal material." Physical Review A. Vol. 44, No. 4, 1991. pp. 25582569.

33. Crawford, G. P., Steele, L. M., Ondris-Crawford, R., Iannacchione, G. S., Yeager, C. J., Doane, J. W., and Finotello, D., "Characterization of the cylindrical cavities of Anopore and Nuclepore membranes.” Journal of Chemical Physics. Vol. 96, No. 10, 1992. pp. 7788-7796.

34. Bradac, Z., Kralj, S., and Zumer, S., "Molecular dynamics study of nematic structures confined to a cylindrical cavity." Physical Review E. Vol. 58, No. 6, 1998. pp. 7447-7454.

35. Crawford, G. P., Yang, D. K., Zumer, S., Finotello, D., and Doane, J. W., "Ordering and SelfDiffusion in the First Molecular Layer at a Liquid-Crystal-Polymer Interface." Physical Review Letters. Vol. 66, No. 6, 1991. pp.723-726. 
36. Jin, T., Zalar, B., Lebar, A., Vilfan, M., Zumer, S., and Finotello, D., “Anchoring and structural transitions as a function of molecular length in confined liquid crystal." The European Physical Journal E. Vol. 16, No. 2, 2005. pp 159-165.

37. Takeuchi, K., "A Numerical Method for Solving the Neutron Transport Equation in Finite Cylindrical Geometry." Journal of Nuclear Science and Technology. Vol. 6, No. 8, 1969. pp. 466473.

38. Kim, J. H., Acharya, B. R., Agra, D. M., and Kumar, S., "Thermal Stability of Liquid Crystal Alignment Layers Prepared by In-Site Ultra-Violet Exposure during Imidization of Polyimide." The Japan Journal of Applied Physics. Part 1, No 4A, 2003. pp.2381-2386.

39. Elias, A. L., Harris, K. D., Bastiaansen, C. W. M., Broer, D. J., and Brett, M. J., "Photopatterned liquid crystalline polymers for microactuators." Journal of Materials Chemistry. Vol. 16, No. 28, 2006. pp. 2903-2912.

40. Sousa, M. E., Broer, D. J., Bastiaansen, C. W. M., Freund, L. B., and Crawford, G. P., "Isotropic "Islands" in a Cholestric "Sea": Patterned Thermal Expansion for Responsive Surface Topologies.” Advanced Materials. Vol. 18, No. 14, 2006. pp. 1842-1845.

41. Mol. G. N., Harris, K. D., Bastiaansen, C. W. M., Broer, D. J., “Thermo-Mechanical Responses of Liquid-Crystal Networks with Splayed Molecular Organization.” Advanced Materials. Vol. 15, No. 2, 2005. pp. 1155-1159.

42. Evans, B. A., Shields, A. R., Lloyd-Carroll, R., Washburn, S., Falvo, M. R., Superfine, R., "Magnetically Acutated Nanorod Arrays as Biomimetic Cilia." Nano Letters. Vol. 7, No.5, 2007. pp. 1428-1434.

43. Dierking, I., and San, S. E., "Magnetically steered liquid crystal-nanotube switch.” Applied Physics Letters. 87, 2005. pp. 233507.1-233507.3.

44. Clark, N. A., and Lagerwall, S. T., "Sub-microsecond bistable electro-optic switching in liquid crystals." Applied Physics Letters. Vol. 36, No. 11, 1980. id. 899.

45. . Juodkazis, S., Shikata, M., Takahashi, T., Matsuo, S., and Misawa, H., "Fast optical switching by a laser-manipulated microdroplet of liquid crystal." Applied Physics Letters. Vol. 74, No. 24, 1999. pp. 3627-3629. 
46. Wood, T. A., Gleeson, H. F., Dickinson, M. R., and Wright, A. J., "Mechanisms of optical angular momentum transfer to nematic liquid crystalline droplets." Applied Physics Letters. Vol. 84, No. 21, 2004. pp. 4292-4294.

47. Wu, K. J., Chu, K. C., Chao, C. Y., Chen Y. F., Lai, C. W., Kang, C. C., Chen, C. Y., and Chau, P. T., "CdS Nanorods Imbedded in Liquid Crystal Cells for Smart Optoelectronic Devices.” Nano Letters. Vol. 7, No. 7, 2007. pp. 1908-1913.

48. van der Zande, B. M. I., Koper, G. J., and Lekkerkerker, H. N. K., “Alignment of Rod-Shaped Gold Particles by Electric Fields.” Journal of Physical Chemistry B. Vol. 103, 1999. pp. 57545760.

49. Sit, J, C., Broer, D. J., and Brett, M J., "Liquid Crystal Alignment and Switching in Porous Chiral Thin Films.” Advanced Materials. Vol. 12, No. 5, 2000. pp.371-373.

50. Kalkar, A. K., Kunte, V. V., and Deshpande, A. A., "Electro-optic Studies on Polymer-Dispersed Liquid Crystal Composite Films. I. Composites of PVB-E7.” Journal of Applied Polymer Science. Vol. 74, No. 14, 1991. pp. 3485-3491.

51. Cupelli, D., Macchione, M., Nicoletta, F. P., Fiplo, G. D., and Chidichimo, G., "Electrically induced changes in polymer dispersed liquid crystals." Applied Physics Letters. Vol. 76, No. 20, 2000. id. 2856.

52. Mei, E., and Higgins, D. A., "Nanometer-scale resolution and depth discrimination in near-field optical microscopy studies of electric-field-induced molecular reorientation dynamics." Journal of Chemical Physics. Vol. 112, No.18, 2000. pp. 7839-7847.

53. Costa, I., Almeida, P. J., Filip, D., Figueirinhas, J. L., and Godinho, M. H., “Tunable topographical cellulose matrices for electro-optical liquid crystal cells." Opto-Electronics Review. Vol. 14, No. 4, 2006. pp. 299-303.

54. Lucchetta, D. E., Karapinar, R., Manni, A., and Simoni, F., "Phase-only modulation by nanosized polymer-dispersed liquid crystals." Journal of Applied Physics. Vol. 91, No. 9, 2002. pp. 60606065 .

55. Vennes, M., Martin, S., Gisler, T., and Zentel, R., “Anisotropic Particles from LC Polymers for Optical Manipulations.” Macromolecules. Vol. 39, No. 5, 2006. pp. 8326-8333.

56. Garces-Chavez, V., McGloin, D., Summers, M. D., Fernandez-Nieves, A., Spalding G. C., Cristobal, G., and Dholakia, K., "The reconstruction of optical angular momentum after distortion in amplitude, phase and polymerization." Journal of Optics A: Pure and Applied Optics. Vol. 6, No. 5, 2004. pp. S235-S238. 
57. Amimori, I., Eakin, J. N., Qi, J., Skacej, G., Zumer, S., and Crawford, G. P., "Surface induced orientational order in stretched nanoscale-size polymer dispersed liquid crystal droplets." Physical Review E. Vol. 71, 2005. pp. 031702.1-031702.11.

58. Nicoletta, F. P., Filpo, G. D., Cupelli, D., Macchione, M., and Chidichimo, G., "Orientation control of liquid crystal droplets dispersed in a polymer matrix." Applied Physics Letters. Vol. 79, No. 26, 2001. id.4325.

59. Amundson, K., "Electro-optic properties of a polymer-dispersed liquid crystal film: Temperature dependence and phase behavior." Physical Review E. Vol. 53, No. 3, 1996. pp.2412-2422.

60. Han, J. W., Kang, T. J., and Park, G., "Effects of Composition, Curing-Time, and Temperature on the Electro-optical Characteristics of Polymer-Dispersed Liquid Crystal Films." Journal of the Korean Physical Society. Vol. 36, No. 3, 2000.

61. Loiko, V. A., Konkolovich, A. V., and Miskevich, A. A., "Order parameter of elongated liquid crystal droplets: The method of retrieval by the coherent transmittance data." Physical Review E. Vol. 74, 2006. id. 031704.

62. Vicari, L., "Electro-optic phase modulation by polymer dispersed liquid crystals." Journal of Applied Physics. Vol. 81, No. 10, 1997. pp.6612-6615.

63. Kosc, T. Z., Jacobs, S. D., and Lambropoulos, J. C., "Polymer cholesteric liquid-cystal flake reorientation in an alternating-current electric field." Journal of Applied Physics. Vol. 98, 2005. pp. 6612-6615.

64. Nicoletta, F. P., Filpo, G. D., Lanzo, J., and Chidichimo, G., "A method to produce reverse-mode polymer-dispersed liquid crystal shutters.” Applied Physics Letters. Vol. 74, No. 26, 1999. id. 3945.

65. Olenik, D., Copic, M., Sousa, M. E., and Crawford, G. P., "Optical retardation of in-plane switched polymer-dispersed liquid crystals.” Journal of Applied Physics. Vol. 100, 2006. pp.033515-0033515-7.

66. Raina, K. K., Kumar, P., and Malik, P., "Morphological control and polarization switching in polymer dispersed liquid crystal materials and devise." Bulletin of Material Science. Vol. 29, No. 6, 2006. pp.599-603. 
67. Teixeira, P. I. C., and Mulder, B. M., "Cell dynamics model of droplet formation in polymerdispersed liquid crystals.” Physical Review E. Vol. 53, No. 2, 1996. pp.1805-1815.

68. Cairns, D. R., Genin, G. M., Wagoner, A. J., Briant, C. L., and Crawford, G. P., "Amplified strain-rate dependence of deformation in polymer-dispersed liquid crystal materials." Applied Physics Letters. Vol. 75, No. 13, 1999. id. 1872.

69. Carter, S. A., LeGrange, J. D., White, W., Boo, J., and Wiltzius, P., "Dependence of the morphology of polymer dispersed liquid crystals on the UV polymerization process." Journal of Applied Physics. Vol. 81, No. 9, 1997. pp. 5992-5999.

70. Geary, J. M., Goodby, J. W., Kmetz, A. R., and Patel, J. S., "The mechanism of polymer alignment of liquid crystal materials.” Journal of Applied Physics. Vol. 62, No. 10, 1987. pp. 4100-4108.

71. Drzaic, P. S., "Polymer dispersed nematic liquid crystal for large area displays and light valves." Journal of Applied Physics. Vol. 60, No. 6, 1986. pp. 2142-2148.

72. Xu, C., Wang, Q., Xu, H., Xie, S., and Yang, Z., "General synthesis of hollow composite ellipsoids.” Colloid Polymer Science. Vol. 285, No. 13, 2007. pp. 1471-1478.

73. Le, Y., Yin, Y., and Xia, Y., "Preparation and Characterization of Micrometer-Sized "Egg Shells"." Advanced Materials. Vol. 13, No. 4, 2001. pp. 271-274.

74. Mukherjee, G. S., "Modification of Poly(vinyl Alcohol) for improvement of mechanical strength and moisture resistance." Journal of Material Science. Vol. 40, No. 7, 2005. pp. 3017-3019.

75. "Wing Morphing of The Swift Could Inspire New Aircraft Designs”, Science Daily, (2007).

76. "Mission Adaptive Compliant Wing (MACW)" FlexSys Inc. Simple Engineered to Flex.

77. "Wing Morphing and Fuel-Efficient Planes", Machine Design.com, (2008).

78. Khatavkar, V. V., Anderson, P. D., den Tooder, J.M.J., and Meijer, H.R.H., "Active micromixer based on artificial cilia.” Physics of Fluids. Vol. 19, No. 8, 2007. pp. 083605.1- 083605.13.

79. Lu, L. H., Ryu, K. S., and Liu, C., "A magnetic microstirrer and array for microfluidic mixing." Journal of Mircoelectromechanical Systems. Vol. 11, No. 5, 2002. pp. 462. 
80. Oddy, M. H., Santiago, J. G., and Mikkelsen, J. C., "Electrokinetic instability micromixing." Analytical Chemistry. Vol. 73, No. 24, 2001.pp. 5822.

81. Liu, R. H., Yank, J., Pindere, M. Z., Athavale, M., and Grodzinski, P., "Bubble-induced acoustic micromixing." Lab on a Chip. Vol. 2, No. 151, 2002.

82. Yang, Z., Goto, H., Matsumoto, M., and Maeda, R., "Active Micromixer for microfluidics systems using lead-zirconate-titanate (PZT) generated ultra-sonic vibration." Electrophoresis. Vol. 2, No. 116, 2000.

83. MacDonald, R., "Liquid Crystal Group.” 1998. Optical Institute of the Technical University of Berlin. 2008. <http://moebius.physik.tu-berlin.de/lc/ldc.html> 